\title{
Observation and modelling of main sequence star chromospheres
}

\section{High resolution observations of $\mathrm{M}$ and $\mathrm{K}$ dwarf chromospheric lines}

\author{
E. R. Houdebine ${ }^{1}$, K. Junghans ${ }^{2}$, M. C. Heanue ${ }^{3}$, and A. D. Andrews ${ }^{2}$ \\ 125 Rue du Dr. Laulaigne, 49670 Valanjou, France \\ e-mail: eric_houdebine@yahoo.fr \\ 2 Armagh Observatory, College Hill, BT61 9DG Armagh, N. Ireland \\ ${ }_{3}$ Dublin Institute for Advanced Studies, Dunsink Observatory, Castleknock, Dublin 15, Ireland \\ Received 14 November 2008 / Accepted 10 May 2009
}

ABSTRACT

\begin{abstract}
Aims. We report on high resolution observations of chromospheric lines for $\mathrm{M}$ and $\mathrm{K}$ dwarfs.
Methods. We observed up to 13 spectral lines per star: the $\mathrm{H}_{\alpha}, \mathrm{H}_{\beta}, \mathrm{H}_{\epsilon}, \mathrm{Pa}_{\epsilon}, \mathrm{Pa}_{8}$, CaII H, CaII IR triplet, CaI $6572 \AA$, NaI $\mathrm{D}_{1} / \mathrm{D}_{2}$ and $\mathrm{HeI}_{3}$ lines. We observed two dMe stars, one $\mathrm{dM}(\mathrm{e})$ star, $5 \mathrm{dM}$ stars and $3 \mathrm{dK}$ stars.

Results. We observed a self-reversal in the emission core of the CaII $\mathrm{H}$ line for the brightest stars only, indicating a rather optically thick region of formation.

We present original spectra of the NaI doublet and the CaII IR triplet for active dMe stars and less active dM stars. Core emission is detected in the Sodium lines and the CaII IR triplet lines for the most active M dwarf AU Mic. We investigate the difference spectra between active $\mathrm{dMe}$ stars and $\mathrm{dM}$ stars and show that these provide interesting new constraints for the NLTE-radiation transfer modelling of the chromospheres.

In our sample, emission $\mathrm{H}_{\alpha}$ profiles have a rather homogeneous $F W H M$ of about $1.5 \AA$. This, according to our previous modelling, can be interpreted as the signature of a rather constant temperature break in the chromosphere. We found that one of our targets (MCC 332) is a binary with a faint but active $\mathrm{H}_{\alpha}$ emission component.

For the first time we detect the $\mathrm{Pa}_{\epsilon}$ line for six dwarfs. It appears as weak absorption with possible weak wing emission in AU Mic. The region of the $\mathrm{Pa}_{8}$ line was observed but the line was not detected.
\end{abstract}

Key words. stars: late-type - stars: activity - stars: chromospheres - stars: magnetic fields

\section{Introduction}

High levels of magnetic activity in main sequence stars have manifested themselves as flares (e.g. Cristaldi \& Rodonó 1970) and chromospheric emission lines (e.g. Vaughan et al. 1981; Giampapa et al. 1982; Rutten et al. 1989). Such phenomena are also observed in pre-main sequence stars (e.g. Shara et al. 1997) and RS CVn systems (e.g. Dempsey et al. 2001; Osten et al. 2003). The most notable chromospheric lines, that serve as stellar classification criteria are the Balmer $\mathrm{H}_{\alpha}$ line and the CaII resonance doublet. Stars showing $\mathrm{H}_{\alpha}$ in emission were classified as emission line stars. For the most active red dwarfs, the chromospheric density is so high that it drives a number of lines into emission (e.g. Cram \& Mullan 1979; Giampapa et al. 1982; Cram \& Mullan 1985) and also affects the formation of usually photospheric lines (e.g. Houdebine \& Doyle 1995, hereafter Paper IV; Houdebine 2009a, hereafter Paper X).

High resolution observations of chromospheric lines from the near-UV to IR wavelengths are essential to understand and constrain the chromospheric phenomena. Such observations were used to constrain the properties of the chromosphere by means of NLTE-radiative transfer modelling in main-sequence stars. For instance, the $E W$ of the CaII resonance lines are often used as a diagnostic of the level of magnetic activity

\footnotetext{
* Based on observations collected at the European Southern Observatory, La Silla, Chile.
}

(e.g. Houdebine \& Stempels 1997, hereafter Paper VI). The $F W H M$ of these lines is known to correlate with the stellar luminosity, known as the Wilson-Bappu relationship (e.g. Wilson \& Bappu 1957, Elgaroy et al. 1999), and appears to be a diagnostic of the chromospheric mass column density (Ayres 1979). The CaII $\mathrm{K}$ to $\mathrm{CaII} \mathrm{H}$ flux ratio seems a good diagnostic of the mid-chromosphere optical depth in M dwarfs (Paper VI). $\mathrm{H}_{\alpha}$ is often used to detect the highest activity stars (e.g. Worden et al. 1981; Herbst \& Layden 1987; Hawley et al. 1996) and as a diagnostic of the upper chromosphere (e.g. Cram \& Mullan 1979, 1985; Houdebine \& Doyle 1994a,b, hereafter Papers I and II; Houdebine et al. 1995, hereafter Paper III).

There has been several efforts to model the spectral signatures of $\mathrm{dM}$ and $\mathrm{dK}$ stars. Some pioneering work was carried out by Cram \& Mullan (1979): they found important properties of the chromosphere of dM stars, namely, the $\mathrm{H}_{\alpha}$ line is weak when the chromospheric pressure is low, then the absorption increases as the pressure increases, and the line eventually goes into emission for the largest pressures. They also found that in dMe stars, the source functions of the Balmer lines are collisionally controlled, whereas in lower pressure dM stars they are photoionization controlled. Kelch et al. (1979) attempted to model $\mathrm{H}_{\alpha}$ observations of EQ Vir and 61 Cyg B with semi-empirical models. The first real proper fits to observations were carried out by Giampapa, Worden \& Linsky (1982). They managed to reproduce rather well the CaII $\mathrm{K}$ line observations of three $\mathrm{dMe}$ and two dM stars. They found that the lower chromospheric 
temperature gradient is similar for all the $\mathrm{M}$ dwarf stars. Cram \& Mullan (1985) found that strong $\mathrm{H}_{\alpha}$ absorption profiles were indeed evidence for the presence of chromospheres in $\mathrm{M}$ dwarfs. Cram \& Giampapa (1987) calculated the $\mathrm{H}_{\alpha}$ and CaII line profiles assuming a slab chromosphere with varying parameters. They found the interesting relation between the $\mathrm{H}_{\alpha}$ equivalent width and the CaII $\mathrm{K}$ equivalent width, that was later confirmed by calculations of grids of model chromospheres.

Subsequently, a substantial amount of work was carried out by the Armagh group (Houdebine 1990; Panagi 1990; Houdebine \& Panagi 1990; Houdebine \& Doyle 1994a,b; Doyle et al. 1994; Houdebine \& Doyle 1995a; Houdebine et al. 1995; Houdebine \& Doyle 1995b; Lanzafame 1995; Lanzafame \& Byrne 1995; Houdebine et al. 1996; Short et al. 1997; Andretta et al. 1997; Short \& Doyle 1998a,b; Jevremovic et al. 2000). Houdebine \& Doyle (1994a,b, Papers I and II thereafter) studied in detail the modelling of the Balmer lines and the Lyman/Balmer flux ratio in the dM1e star AU Mic. They proposed for the first time a model that could reproduce these observations.

Doyle et al. (1994) and Houdebine \& Doyle (1995b) proposed chromospheric models for the other end of the activity range: the basal chromosphere $\mathrm{M}$ dwarfs for which the contribution from plages can be neglected. On the basis of the models of the two extremes of the activity range, dM1e stars and dM1 basal stars, Houdebine et al. (1995) built the first grid of model atmospheres that cover the whole range of activity for dM1 stars.

Lanzafame (1995) and Lanzafame \& Byrne (1995) analyzed several spectral lines of the dMe star G1 182. Lanzafame (1995) produced two grids of model chromospheres with a high column mass at the transition region between $\log (M)=-4.0$ to $\log (M)=-4.15$. They could reproduce partly the $\mathrm{H}_{\alpha}$ line profile but the self-reversal of the models was too strong.

Short et al. (1997) focused their analysis on the CaI $4227 \AA$ line. Andretta et al. (1997) investigated the formation of the NaI $\mathrm{D} 1 / \mathrm{D} 2$ resonance lines in $\mathrm{dM}$ stars. They found that these lines are interesting chromospheric diagnostics and that it is necessary to include a proper treatment of the background opacities. Short \& Doyle (1998a) modelled the $\mathrm{H}_{\alpha}$ line and the $\mathrm{Pa}_{\beta}$ line in the dM1e star AU Mic. They could reproduce separately, but not simultaneously the $\mathrm{H}_{\alpha}$ line and the $\mathrm{Pa}_{\beta}$ line. Again their models assumed a uniform chromosphere. Short \& Doyle (1998b) attempted to model simultaneously the $\mathrm{H}_{\alpha}$ line and the NaI D lines. The profiles cannot be simultaneously fitted for all but one of their stars. Jevremovic et al. (2000) studied the effects of micro-turbulent velocities on the formation of the $\mathrm{H}_{\alpha}$ line, the $\mathrm{CaII}$ resonance lines and the $\mathrm{NaI}$ resonance lines. They found that this chromospheric parameter has an important influence on the formation of these lines.

Some other authors have also contributed to the progress of semi-empirical modelling of dM stellar chromospheres. Mauas \& Falchi (1994) modelled the chromosphere of AD Leonis assuming a homogeneous chromosphere. Mauas et al. (1997) developed models for two dM stars considered as basal, based on the fit of several lines. Houdebine \& Stempels (1997) proposed an improved grid of 30 model chromospheres for dM1 stars, from basal to the most active dM1e stars. Baranovskii et al. (2001) modelled three dMe stars on the basis of Balmer line observations. Busa et al. (2001) re-investigated in detail the importance of line blanketing in the formation of several important chromospheric lines in the case of main-sequence dwarfs. Andretta et al. (2005) studied the CaII infrared triplet as a potential stellar activity diagnostic. Osten et al. (2006) derived a model atmosphere above $10000 \mathrm{~K}$ for the dMe star EV Lac.
Walkowicz \& Hawley (2008) analysed the chromospheric spectral signatures of many dM3 stars, in a similar way as in Houdebine \& Stempels (1997). Walkowicz (2008) and Walkowicz \& Hawley (2009) derived models for dM3 stars. They found that for low and intermediate activity stars, only a single component model chromosphere is necessary to reproduce observations, but that active dM3 stars necessitate a twocomponent model chromosphere. Houdebine (2009a, Paper X) proposed the first two-component model chromosphere for a dM1 star: namely the intermediate activity star Gl 205.

Some interesting studies of $\mathrm{dK}$ stars have also been performed: Cuntz et al. (1999) propose theoretical ab-initio models with two components. This was the first study to really propose two-component solutions for the observations: the quiescent region component is heated by acoustic waves, and the active region component, magnetic in character, is heated by longitudinal tube waves. Sim \& Jordan (2005) developed a model for $\epsilon$ Eri (dK2) based on the emission measure distribution of UV lines. They extend their model to lower chromospheric temperatures in a semi-empirical way. They also derive a crude twocomponent model for this star.

Recently, in Paper XI in this series (Houdebine 2009b) we defined a new method to constrain the theoretical profiles of the $\mathrm{H}_{\alpha}$ line and the CaII $\mathrm{H} \& \mathrm{~K}$ resonance lines from the measurements of their equivalent widths. This method is based on the calculations of the grid of models of Paper VI. We showed that, providing that such a grid of model chromospheres is available, one can derive two-component model chromospheres for quiescent and active regions on dM1 stars. In Paper XI, we derived such model chromospheres for nine near-solar metallicity dM1 stars. Many were found to be rather active, with sometimes activity levels close to those found in dM1e stars. Such models are very important because they allow us to understand the properties of quiescent regions as well as active regions on $\mathrm{M}$ dwarfs. Houdebine (2009c, hereafter Paper XII) also developed twocomponent model chromospheres with the same technique for seven dM1e stars.

There are several other spectral lines of great interest such as the $\mathrm{NaI} \mathrm{D}_{1} / \mathrm{D}_{2}$ lines, the CaII IR triplet lines and a large number of faint emission lines in the near-UV (Fuhrmeister et al. 2005). These lines can constrain the chromosphere from the temperature minimum to the transition region. However, most observations were dedicated to the study of the CaII resonance lines or the $\mathrm{H}_{\alpha}$ line. Here, we attempt to gather observations of as many lines as possible for a few stars.

Observations of spectral lines are also the basis for developing NLTE-radiation transfer model chromospheres. The chromospheres of $\mathrm{M}$ dwarfs is still little understood in particular for intermediate activity stars, although some recent work has developed two-component model chromospheres for these stars (Papers XI and XII). In Paper VI, we built a grid of model chromospheres for M1 dwarfs (including AU Mic) and calculated the $\mathrm{H}_{\alpha}$ and $\mathrm{CaII} \mathrm{H} \& \mathrm{~K}$ line profiles, that we successfully compared to high resolution observations. A number of authors built some grids of model atmospheres, but exact models of single stars are few (see references above). This kind of model is essential to give constraints to the grids of model atmospheres (e.g. Paper VI). Both detailed modelling and grids of models are necessary to understand the chromospheric phenomenon. Detailed models give constraints to the grids of models, and from the grids of models we can build two-component models (Papers XI and XII).

Here, we gathered observations of as many lines as possible for a few stars, of spectral types late $\mathrm{dK}$ and $\mathrm{dM}$, in order to have 
the most complete constraints for the purpose of NLTE radiation transfer modelling of the chromosphere. A partial report of these observations can be found in Houdebine (1990). However, this later study did not analyze nor comment on the results. The results for the Paschen lines were not presented either.

\section{Observations and data reduction}

The observations presented below were collected during an observing run at the European Southern Observatory with the $1.4 \mathrm{~m}$ Coudé Auxiliary Telescope equipped with the Coudé Echelle Spectrograph (CES) and a CCD detector. The resolving power was 40000 . Simultaneous photometry was carried out with the $50 \mathrm{~cm}$ telescope and a standard photometer in order to assess the flaring or quiescent state of the stars during observations. Weather conditions at ESO varied from poor to good and as a consequence, telluric water vapor lines can be strong.

Our aim was to collect the most complete set of chromospheric line profiles in high resolution for a few stars with different spectral types and activity levels. We also observed stars with similar spectral types but different levels of activity in order to assess its effects on line profiles, from the photosphere to the transition region. These spectra are used as the basis for the detailed NLTE modelling of their chromosphere. The Balmer line observations for AU Mic were used to develop a model chromosphere for this star (Papers I and II). Some results of the CaII lines and Balmer lines for M1 dwarfs were also compared to the grid of models of Paper VI. We will use the data presented here to build a model chromosphere for each individual star. The stars presented here span from low activity to high activity. The resulting detailed model chromospheres will be used in future studies to develop new grids of model chromospheres. We emphasize that this data is rather complete and can be used to constrain the atmosphere from the photosphere to the lower transition region.

Observations at ESO were obtained over five nights in different spectral regions. This is of the order of the rotation period of the most active dwarfs, which therefore may result in unwanted rotational modulation in the line profiles. Nevertheless, observations indicate so far that such modulation is somewhat weak (e.g. Baliunas 1981). No flares were detected, either in white light or in the line profiles. We therefore assume that the spectra discussed here are representative of the stellar quiescent state.

The spectra were reduced following the standard procedure. Dark exposures, flat-field and Thorium spectra were gathered regularly in order to assess variations in dark counts, flat-field irregularities and wavelength stability. We found that they were quite stable in time. The spectra were flat-field corrected, sky subtracted, and wavelength calibrated using the Thorium-Argon spectra. The wavelength calibration gives typically an accuracy of $\Delta \lambda / \lambda \sim 6 \times 10^{6}$ (i.e. $\sim 0.01 \AA$ at $6500 \AA$ ).

However, we faced some problems when applying the flatfield correction for wavelengths greater than $8000 \AA$, owing to large amplitude interference fringes. Because the light paths of the stellar and flat-field sources are not exactly identical in the spectrograph, the resulting fringe patterns also change slightly. This results in a slightly inadequate flat-field correction with an amplitude of about $5 \%$ of the normalised continuum. We also emphasize that the fringe patterns are much broader than typical photospheric absorption lines. This implies that it does not affect the detection of absorption lines, notably the Paschen lines. We generally found that a reasonably good correction can be achieved (less than $\sigma=5 \%$ in the line profiles and continuum) that is sufficient for the purpose of this study.

\section{Results}

In total, we obtained 71 high resolution spectra for 10 different stars of spectral types late $\mathrm{dK}$ and $\mathrm{dM}$. The summary of our observations is given in Table 1 . The spectra are shown from Fig. 1 to 9: CaII H \& $\mathrm{H}_{\epsilon}$ in Fig. $1, \mathrm{H}_{\beta}$ in Fig. 2, NaI D1 \& D2 in Fig. 3, $\mathrm{H}_{\alpha}$ in Fig. 4, CaII $8498 \AA$ in Fig. 5, CaII $8543 \AA$ in Fig. 6, CaII $8662 \AA$ in Fig. 7, $\mathrm{Pa}_{\epsilon}$ in Fig. 8 and $\mathrm{Pa}_{8}$ in Fig. 9. We also show the difference spectra AU Mic-Gl 887 in Fig. 10 and AU MicGl 908 in Fig. 11. In our diagrams, all spectra were normalized to the continuum (we define the continuum here as the upper boundary delineated by the photospheric lines). A constant shift was added in the $Y$-axis for better clarity. We show the zero level for each spectrum as a dotted-dashed line. We discuss below our observations and the properties of the chromosphere for each star separately.

We present here the first observations and detections of the $\mathrm{Pa}_{\epsilon}$ line in 6 stars (Fig. 8). The region of $\mathrm{Pa}_{8}$ was also observed for two stars but this line was not detected (Fig. 9). We also present original observations of the $\mathrm{NaI}$ doublet (Fig. 3) and the CaII IR triplet lines (Figs. 5 to 7).

For the $\mathrm{Pa}_{\epsilon}$ line, the spectra are heavily contaminated by telluric absorption features. We observed a standard star, HD 8946 (spectral type A0), in order to remove these telluric spectral features. The spectra were all normalized to the continuum, and the spectrum of HD 8946 was subtracted from the spectra of our target stars. This yields the line profile of $\mathrm{Pa}_{\epsilon}$ which is not present in HD 8946. We then added a constant (unity) to the subtracted spectra in order to mimic the background continuum and measure the equivalent widths. The resulting spectra are shown in Fig. 8.

It is difficult to measure spectral line equivalent widths for late $\mathrm{dK}$ and $\mathrm{dM}$ stars because of the uncertainty in the level of the adjacent continuum. Indeed, the background continuum contains numerous faint absorption photospheric features. In order to measure the equivalent widths, we defined two regions $3 \AA$ wide on each side of the spectral line. We averaged the flux in these spectral regions that we define as the continuum level. The spectral line flux is then integrated between these two regions. The equivalent width is the ratio of this flux to the average flux in the two spectral regions on either side of the line.

\subsection{Gl 488}

Cenarro et al. (2006) classified this star as an M0.5 dwarf with $T_{\text {eff }}=3750 \mathrm{~K}, \log (g)=4.75$ and $[\mathrm{M} / \mathrm{H}]=0.10$. For Gl 488 , we observed the $\mathrm{H}_{\alpha}$ line, the $\mathrm{Ca}$ I 6572 and the $\mathrm{Na}$ I doublet. We measured an $\mathrm{H}_{\alpha}$ line equivalent width of $0.588 \pm 0.05 \AA$. Wilson \& Wooley (1970) recorded spectrograms of Gl 488 in the $\mathrm{Ca}$ II $\mathrm{H}$ and $\mathrm{K}$ lines. However they only performed visual estimates of the intensity of these lines against a reference star 61 Cygni. The line intensity was +1 on their scale which means that it is slightly stronger than the reference 61 Cygni. Stauffer \& Hartmann (1986) observed the $\mathrm{H}_{\alpha}$ line and measured an equivalent width of $0.59 \AA$. This is the same as our measurement of $0.588 \pm 0.05 \AA$ for this line.

Mathioudakis \& Doyle (1992) measured the flux in the $\mathrm{Mg}$ II $\mathrm{h} \& \mathrm{k}$ lines observed with IUE. They inferred a surface flux of $\log F_{\mathrm{MgII}}=5.64 \mathrm{ergs} \mathrm{s}^{-1} \mathrm{~cm}^{-2}$. This is relatively high compared to other values inferred for $\mathrm{dM}$ stars and is comparable to values observed for dMe stars. Similarly, Rutten et al. (1989) report a surface flux $\log F_{\text {CaII }}=5.58 \mathrm{ergs} \mathrm{s}^{-1} \mathrm{~cm}^{-2}$ in the Ca II H \& K lines. Wright et al. (2004) observed a CaII S 
Table 1. Summary of ESO observations and line profile parameters.

\begin{tabular}{|c|c|c|c|c|c|c|c|}
\hline $\begin{array}{c}\text { Star } \\
\text { Parameters } \\
\end{array}$ & $\begin{array}{l}\text { Spectral } \\
\text { line }\end{array}$ & $\begin{array}{l}\text { UT } \\
\text { start }\end{array}$ & $\begin{array}{l}\text { Duration } \\
(\mathrm{s})\end{array}$ & $\begin{array}{l}\lambda \\
\AA \\
\AA\end{array}$ & $\begin{array}{c}F W H M \\
\AA \\
\end{array}$ & $\begin{array}{l}E W \\
\mathrm{m \AA}\end{array}$ & $\begin{array}{c}\text { Photometry } \\
U \text {-band }\end{array}$ \\
\hline Gl 488 & $\mathrm{H}_{\alpha}$ & $23: 57: 35 / 21$ & 1200 & 6563.532 & 0.95 & $588 \pm 50$ & $\mathrm{Y}$ \\
\hline $\mathrm{dM} 0.5$ & CaI 6572 & $23: 06: 35 / 21$ & 1200 & 6573.56 & - & $281 \pm 30$ & $\mathrm{Y}$ \\
\hline$v=8.51$ & $\mathrm{NaI} \mathrm{D}_{1,2}$ & $01: 12: 30 / 21$ & 900 & - & - & $11780 \pm 500$ & Y \\
\hline $\begin{array}{c}\text { Gl } 526 \\
\text { dM4 } \\
v=8.5\end{array}$ & CaII IRT 8542 & 23:54:04/19 & 350 & - & - & $1920 \pm 200$ & $\mathrm{~N}$ \\
\hline Gl 588 & $\mathrm{H}_{\alpha}$ & $02: 18: 19 / 21$ & 1800 & 6563.524 & 0.71 & $455 \pm 50$ & $\mathrm{Y}$ \\
\hline $\mathrm{dM} 4$ & $\mathrm{H}_{\beta}$ & $23: 03: 21 / 23$ & 4800 & - & - & - & $\mathrm{N}$ \\
\hline \multirow[t]{8}{*}{$v=10.1$} & $\mathrm{H}_{\epsilon}$ & $02: 30: 59 / 23$ & 2700 & - & - & - & $\mathrm{Y}$ \\
\hline & CaI 6572 & $23: 16: 29 / 20$ & 1800 & 6573.53 & - & $419 \pm 30$ & $\mathrm{Y}$ \\
\hline & CaII H & $02: 30: 59 / 23$ & 2700 & *3968.94 & $* 0.22$ & $*-346 \pm 10$ & $\mathrm{~N}$ \\
\hline & CaII IRT 8498 & $02: 47: 30 / 22$ & 1800 & 8499.000 & 0.44 & $517 \pm 50$ & $\mathrm{Y}$ \\
\hline & CaII IRT 8542 & $01: 30: 23 / 20$ & 900 & 8543.050 & 0.69 & $1234 \pm 200$ & $\mathrm{~N}$ \\
\hline & CaII IRT 8662 & 04:59:16/22 & 1800 & 8663.179 & 0.72 & $1155 \pm 200$ & $\mathrm{Y}$ \\
\hline & $\mathrm{NaI} \mathrm{D}_{1,2}$ & $23: 16: 29 / 20$ & 1800 & - & - & $7330 \pm 500$ & $\mathrm{Y}$ \\
\hline & $\mathrm{Pa}_{\epsilon}$ & - & - & 9547.31 & - & $105 \pm 10$ & - \\
\hline Gl 664 & $\mathrm{H}_{\alpha}$ & $02: 59: 33 / 21$ & 600 & 6562.911 & 1.04 & $640 \pm 50$ & $\mathrm{Y}$ \\
\hline $\mathrm{dK} 5$ & $\mathrm{H}_{\beta}$ & $05: 50: 21 / 23$ & 900 & - & - & $\sim 410 \pm 50$ & $\mathrm{~N}$ \\
\hline \multirow[t]{6}{*}{$v=6.34$} & CaI 6572 & 02:33:31/19 & 1340 & 6572.90 & - & $145 \pm 10$ & $\mathrm{~N}$ \\
\hline & CaII IRT 8498 & $02: 20: 52 / 22$ & 900 & $\sim 8498.19$ & 1.42 & $1070 \pm 100$ & $\mathrm{Y}$ \\
\hline & CaII IRT 8542 & $04: 33: 54 / 19$ & 1260 & 8542.160 & 3.41 & $3030 \pm 200$ & $\mathrm{~N}$ \\
\hline & CaII IRT 8662 & $05: 34: 24 / 22$ & 900 & 8662.323 & 2.20 & $2120 \pm 200$ & $\mathrm{Y}$ \\
\hline & $\mathrm{NaI} \mathrm{D}_{1,2}$ & $02: 33: 31 / 19$ & 1340 & - & - & $6800 \pm 500$ & $\mathrm{~N}$ \\
\hline & $\mathrm{Pa}_{\epsilon}$ & - & - & 9546.23 & - & $84 \pm 10$ & - \\
\hline G1 729 & $\mathrm{H}_{\alpha}$ & 03:14:42/21 & 1800 & 6562.392 & 1.23 & $-1230 \pm 100$ & Y \\
\hline $\mathrm{dM} 4.5 \mathrm{e}$ & $\mathrm{H}_{\beta}$ & $04: 05: 47 / 23$ & 3000 & 4861.050 & 0.705 & $-1736 \pm 100$ & $\mathrm{~N}$ \\
\hline \multirow[t]{6}{*}{$v=10.6$} & CaI 6572 & 08:04:34/22 & 1800 & 6572.42 & - & $489,5 \pm 50$ & $\mathrm{Y}$ \\
\hline & CaII IRT 8498 & $03: 24: 44 / 22$ & 1800 & $\sim 8497.58$ & - & $\sim 385 \pm 100$ & $\mathrm{Y}$ \\
\hline & CaII IRT 8542 & $07: 01: 32 / 21$ & 2400 & 8541.810 & 1.98 & $1340 \pm 100$ & $\mathrm{Y}$ \\
\hline & CaII IRT 8662 & $04: 22: 56 / 22$ & 1800 & 8661.431 & 1.05 & $1055 \pm 100$ & $\mathrm{Y}$ \\
\hline & $\mathrm{NaI} \mathrm{D}_{1,2}$ & 08:04:34/22 & 1800 & - & - & $8120 \pm 500$ & $\mathrm{Y}$ \\
\hline & $\mathrm{HeI} \mathrm{D}_{3}$ & 08:04:34/22 & 1800 & $\sim 5875.35$ & - & $\sim-114 \pm 10$ & $\mathrm{Y}$ \\
\hline AU Mic & $\mathrm{H}_{\alpha}$ & $04: 57: 26 / 21$ & 900 & 6562.306 & 1.415 & $-2466 \pm 100$ & Y \\
\hline (Gl 803) & $\mathrm{H}_{\beta}$ & $05: 24: 01 / 23$ & 1200 & 4860.860 & 0.793 & $-1912 \pm 100$ & $\mathrm{~N}$ \\
\hline dM1e & $\mathrm{H}_{\epsilon}$ & $07: 59: 32 / 23$ & 1800 & 3969.98 & $\sim 0.44$ & $-1081 \pm 100$ & $\mathrm{~N}$ \\
\hline \multirow[t]{7}{*}{$v=8.61$} & CaI 6572 & 08:45:02/19 & 1200 & 6572.30 & - & $427 \pm 50$ & $\mathrm{~N}$ \\
\hline & CaII H & $07: 59: 32 / 23$ & 1800 & *3968.20 & 0.31 & $-4554 \pm 200$ & $\mathrm{~N}$ \\
\hline & CaII IRT 8498 & 09:53:40/22 & 600 & ${ }^{*} 8497.400$ & ${ }^{*} 0.45$ & $\begin{array}{c}*-381 \pm 50 \\
420 \pm 50\end{array}$ & $\mathrm{~N}$ \\
\hline & CaII IRT 8542 & 05:48:00/19 & 2700 & *8541.429 & ${ }^{*} 0.520$ & $\begin{array}{l}*-446 \pm 50 \\
1320 \pm 200\end{array}$ & $\mathrm{~N}$ \\
\hline & $\mathrm{NaI} \mathrm{D}_{1,2}$ & 09:16:42/19 & 1200 & - & - & $\begin{array}{c}*-62.9 \pm 10,-32.4 \pm 10 \\
7982 \pm 500\end{array}$ & $\mathrm{~N}$ \\
\hline & $\mathrm{HeI} D_{3}$ & 09:16:42/19 & 1200 & 5875.271 & 0.551 & $129 \pm 10$ & $\mathrm{~N}$ \\
\hline & $\mathrm{Pa}_{\epsilon}$ & - & - & 9545.34 & - & $173 \pm 10$ & - \\
\hline
\end{tabular}


Table 1. continued.

\begin{tabular}{|c|c|c|c|c|c|c|c|}
\hline $\begin{array}{c}\text { Star } \\
\text { Parameters }\end{array}$ & $\begin{array}{c}\text { Spectral } \\
\text { line } \\
\end{array}$ & $\begin{array}{c}\text { UT } \\
\text { start }\end{array}$ & $\begin{array}{c}\text { Duration } \\
\text { (s) }\end{array}$ & $\begin{array}{l}\lambda \\
\AA \\
\end{array}$ & $\begin{array}{c}F W H M \\
\AA \\
\end{array}$ & $\begin{array}{l}E W \\
\mathrm{~m} \AA\end{array}$ & $\begin{array}{c}\text { Photometry } \\
U \text {-band }\end{array}$ \\
\hline AX Mic & $\mathrm{H}_{\alpha}$ & $04: 01: 37 / 21$ & 600 & 6562.868 & 0.856 & $592 \pm 50$ & $\mathrm{Y}$ \\
\hline (Gl 825) & $\mathrm{H}_{\beta}$ & $05: 05: 32 / 23$ & 900 & - & - & $\sim 200 \pm 50$ & $\mathrm{~N}$ \\
\hline $\mathrm{dK} 7$ & $\mathrm{H}_{\epsilon}$ & $08: 36: 48 / 23$ & 1200 & - & - & $\sim 4.3 \pm 2$ & $\mathrm{~N}$ \\
\hline \multirow[t]{6}{*}{$v=6.67$} & CaI 6572 & $09: 45: 21 / 19$ & 900 & 6572.86 & - & $249 \pm 30$ & $\mathrm{~N}$ \\
\hline & CaII H & $08: 36: 48 / 23$ & 1200 & 3968.515 & 0.33 & $-599 \pm 50$ & $\mathrm{~N}$ \\
\hline & CaII IRT 8498 & $10: 06: 46 / 22$ & 300 & & 1.04 & $975 \pm 100$ & $\mathrm{~N}$ \\
\hline & CaII IRT 8542 & 06:30:13/19 & 600 & 8542.83 & 2.13 & $2460 \pm 200$ & $\mathrm{~N}$ \\
\hline & $\mathrm{NaI} \mathrm{D}_{1,2}$ & $09: 45: 21 / 19$ & 900 & - & - & $9111 \pm 500$ & $\mathrm{~N}$ \\
\hline & $\mathrm{Pa}_{\epsilon}$ & - & - & 9546.12 & - & $92 \pm 10$ & - \\
\hline Gl 884 & $\mathrm{H}_{\alpha}$ & 09:54:47/21 & 900 & 6562.576 & 0.98 & $605 \pm 50$ & $\mathrm{Y}$ \\
\hline dK5 & $\mathrm{H}_{\epsilon}$ & $10: 28: 04 / 23$ & 1800 & 3969.93 & - & $-1250 \pm 100$ & $\mathrm{~N}$ \\
\hline \multirow[t]{4}{*}{$v=7.89$} & CaI 6572 & & & 6572.55 & - & $235 \pm 30$ & \\
\hline & CaII H & $10: 28: 04 / 23$ & 1800 & 3968.33 & 0.32 & $-968 \pm 50$ & $\mathrm{~N}$ \\
\hline & CaII IRT 8498 & $10: 48: 13 / 22$ & 600 & 8497.907 & 1.56 & $1007 \pm 100$ & $\mathrm{Y}$ \\
\hline & $\mathrm{Pa}_{\epsilon}$ & - & - & 9545.63 & - & $46 \pm 10$ & - \\
\hline Gl 887 & $\mathrm{H}_{\epsilon}$ & 09:03:31/23 & 1200 & - & - & $\sim-110 \pm 30$ & $\mathrm{~N}$ \\
\hline dM0.5 & CaII H & 09:03:31/23 & 1200 & 3968.271 & 0.25 & $-796 \pm 50$ & $\mathrm{~N}$ \\
\hline \multirow[t]{3}{*}{$v=7.36$} & CaII IRT 8498 & 11:01:40/22 & 1030 & 8497.61 & 0.90 & $742 \pm 100$ & $\mathrm{~N}$ \\
\hline & CaII IRT 8542 & $11: 15: 25 / 22$ & 430 & 8541.63 & 2.07 & $2130 \pm 100$ & $\mathrm{~N}$ \\
\hline & $\mathrm{Pa}_{\epsilon}$ & - & - & 9545.58 & - & $97 \pm 20$ & - \\
\hline Gl 908 & $\mathrm{H}_{\alpha}$ & $09: 32: 34 / 21$ & 900 & 6560.550 & 0.691 & $360 \pm 50$ & $\mathrm{Y}$ \\
\hline $\mathrm{dM} 1$ & CaI 6572 & $10: 40: 14 / 19$ & 1300 & 6570.56 & - & $332 \pm 50$ & $\mathrm{~N}$ \\
\hline \multirow[t]{4}{*}{$v=8.98$} & CaII H & 09:28:47/23 & 3300 & 3967.15 & 0.14 & $-188 \pm 30$ & $\mathrm{~N}$ \\
\hline & CaII IRT 8498 & $10: 28: 55 / 22$ & 900 & 8495.148 & 0.50 & $755 \pm 100$ & $\mathrm{~N}$ \\
\hline & CaII IRT 8542 & $07: 57: 16 / 21$ & 1200 & 8539.24 & 0.98 & $1950 \pm 100$ & $\mathrm{Y}$ \\
\hline & $\mathrm{NaI} D_{1,2}$ & 10:40:14/19 & 1300 & - & - & $7590 \pm 500$ & $\mathrm{~N}$ \\
\hline MCC 332 & $\mathrm{H}_{\alpha}$ & 05:19:10/21 & 1200 & - & - & $288 \pm 50$ & $\mathrm{Y}$ \\
\hline $\mathrm{dK} 7 / \mathrm{dM} 3.5(\mathrm{e})$ & $\mathrm{H}_{\beta}$ & 06:09:33/23 & 1800 & - & - & $\sim 271 \pm 40$ & $\mathrm{~N}$ \\
\hline \multirow[t]{7}{*}{$v=9.4$} & $\mathrm{H}_{\epsilon}$ & $06: 52: 24 / 23$ & 3600 & 3969.98 & - & $\sim-248 \pm 30$ & $\mathrm{~N}$ \\
\hline & CaI 6572 & $07: 13: 19 / 22$ & 1800 & 6572.29 & - & $197 \pm 30$ & $\mathrm{Y}$ \\
\hline & CaII H & $06: 52: 24 / 23$ & 3600 & 3968.22 & 0.31 & $-866 \pm 50$ & $\mathrm{~N}$ \\
\hline & CaII IRT 8498 & $10: 15: 26 / 22$ & 600 & 8497.30 & 1.93 & $\sim 931 \pm 200$ & $\mathrm{Y}$ \\
\hline & CaII IRT 8542 & $06: 25: 03 / 21$ & 1500 & *8541.720 & - & $*-57 \pm 20$ & $\mathrm{Y}$ \\
\hline & & & & & & $2494 \pm 200$ & \\
\hline & $\mathrm{NaI} \mathrm{D}_{1,2}$ & $07: 13: 19 / 22$ & 1800 & - & - & $6550 \pm 500$ & $\mathrm{Y}$ \\
\hline
\end{tabular}

* Emission core only.

The total exposure time and start of the observing sequence are given respectively in Cols. 4 and 3. The date of June 1989 is given after the Universal Time. In the last column we indicate when simultaneous photometry in the Johnson $U$-band was obtained with the ESO $50 \mathrm{~cm}$ telescope.

index of 1.793 . The $S$-value is defined by the operation of the Mount Wilson spectrometers, (Duncan et al. 1991) which measure a quotient of the flux in two triangular bandpasses centered on the $\mathrm{H}$ and $\mathrm{K}$ emission cores and two continuum regions on either side. The $\mathrm{H}_{\alpha}$ equivalent width is also large for a dM0.5 star. According to Houdebine \& Stempels (1997; see also Houdebine et al. 1995, Paper III) calculations, this shows that Gl 488 is an intermediate activity star. That is to say, it has an activity level just below that of the dMe stars, when absorption in $\mathrm{H}_{\alpha}$ attains a maximum before going into emission (Houdebine \& Stempels 1997). This agrees with the finding that this star is slightly metal rich (Paper VI). Indeed, in Paper VI we found that activity level correlates with radius in $\mathrm{dM} 1$ stars, and because radius correlates with metallicity (Houdebine 2008, hereafter Paper VII) then activity correlates with metallicity. Therefore, metal rich stars are on average more active than metal poor stars. 

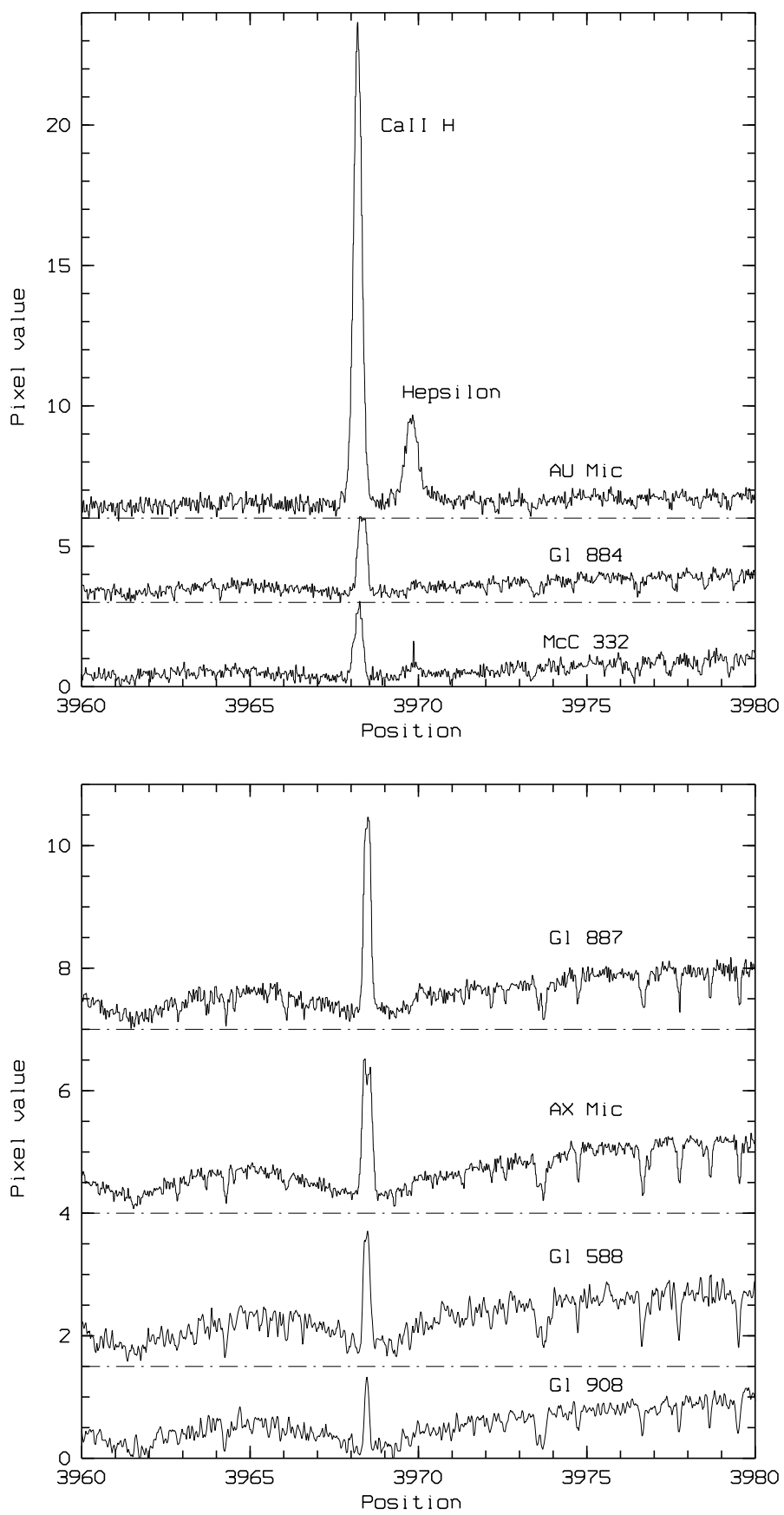

Fig. 1. We show the CaII $\mathrm{H}$ line and the $\mathrm{H}_{\epsilon}$ line in increasing order of the $\mathrm{CaII} \mathrm{H}$ line equivalent width of the emission core. Note that the inner wings progressively disappear and that $\mathrm{H}_{\epsilon}$ is just about detectable for McC 332, Gl 884 and Gl 887.

\subsection{Gl 526}

Gl 526 is an M1 dwarf with $(R-I)_{\mathrm{c}}=1.124, T_{\text {eff }}=3470 \mathrm{~K}, R=$ $0.582 R_{\odot}$ and $[\mathrm{M} / \mathrm{H}]=-0.086$ (Paper VII). It is therefore a small M1 dwarf relative to solar metallicity M1 dwarfs (Paper VII) and is therefore slightly metal-poor. For Gl 526, we observed only the Ca II 8542 line.

Wilson \& Wooley (1970) observed the Ca II H \& K lines and report an activity index of -3 , which implies a very low activity compared to 61 Cygni. Blanco et al. (1974) observed the $\mathrm{Ca}$ II $\mathrm{K}$ line and measured an equivalent width of the core emission of $0.40 \AA$. From this they calculated a surface flux of $\log F_{K}=4.51 \mathrm{ergs} \mathrm{s}^{-1} \mathrm{~cm}^{-2}$. Giampapa et al. (1981) also

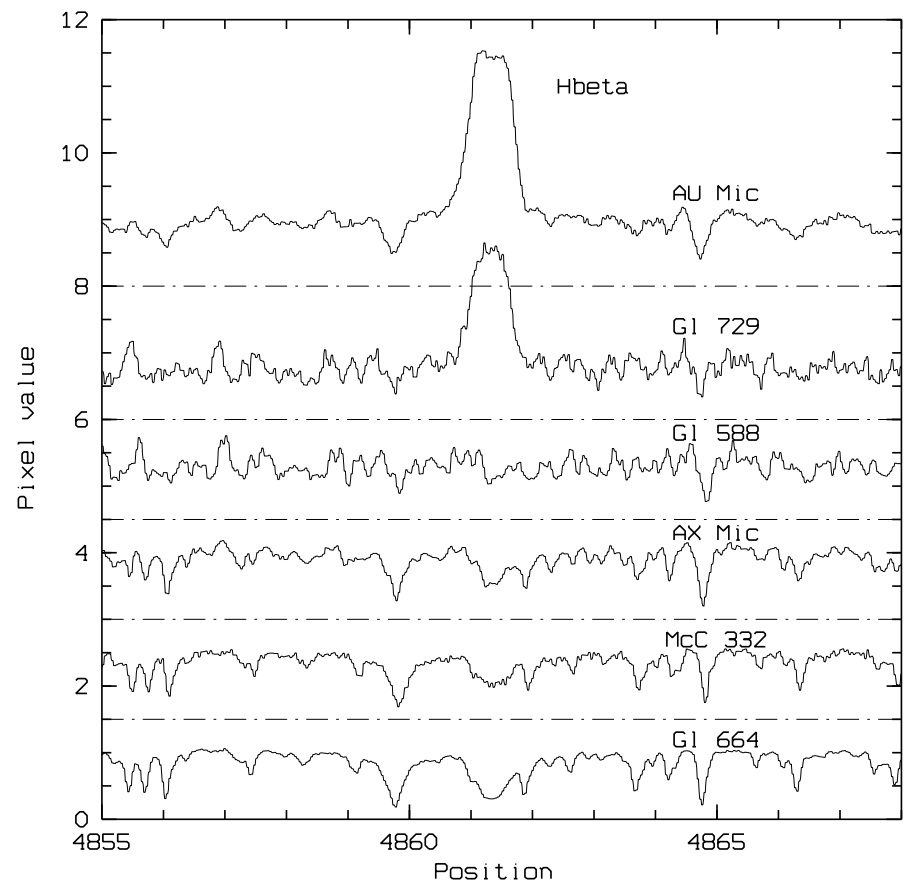

Fig. 2. We show the $\mathrm{H}_{\beta}$ line for six stars.

observed the Ca II H \& $\mathrm{K}$ lines. They calculated excess surface fluxes (the flux from a radiative equilibrium atmosphere was subtracted) of $\log F_{H}=4.23 \mathrm{ergs} \mathrm{s}^{-1} \mathrm{~cm}^{-2}$ and $\log F_{K}=$ $4.26 \mathrm{ergs} \mathrm{s}^{-1} \mathrm{~cm}^{-2}$ which is slightly smaller than the Blanco et al. (1974) flux. They also give the line widths: $F W H M_{H}=$ $0.26 \AA$ and $F W H M_{K}=0.27 \AA$. Cincunegui et al. (2007) found $\log F_{H K}=4.74 \mathrm{ergs} \mathrm{s}^{-1} \mathrm{~cm}^{-2}$. Wright et al. (2004) report a CaII $\mathrm{S}$ index of only 0.787 . Rauscher \& Marcy (2006) give CaII H \& K equivalent widths of $0.44 \AA$ and $0.61 \AA$ respectively. Here, we only observed the CaII $8542 \AA$ line. This line has an average $E W$ of $1.92 \AA$. Therefore, Gl 526 is a low activity slightly metal poor M1 dwarf.

G1 526 was also observed with IUE. Mathioudakis \& Doyle (1989) gave a surface flux for the $\mathrm{Mg}$ II h \& k lines; $\log F_{\mathrm{Mg}}=$ 5.52 , more than an order of magnitude larger than the flux in the $\mathrm{Ca}$ II H \& $\mathrm{K}$ lines. This departs from the nearly one to one correlation found by Panagi \& Mathioudakis (1993) for these lines, and possibly arises from the variability of this star. Tripicchio et al. (1999) observed the Na I doublet and the K I $\lambda 7699$ resonance line and they gave equivalent widths of $0.85 \AA$ and $8.7 \AA$ respectively.

Stauffer \& Hartmann (1986) report an $\mathrm{H}_{\alpha}$ equivalent width of about $0.45 \AA$. Herbst \& Layden (1987) give a very close value of $0.44 \AA$, determined photometrically. Kamper et al. (1997) also measured an $\mathrm{H}_{\alpha}$ equivalent width that is of the same order; $0.49 \AA$. Kraft et al. (1964) measured a $F W H M$ of $0.74 \AA$ for $\mathrm{H}_{\alpha}$. Cutispoto \& Giampapa (1988) also observed spectroscopically the $\mathrm{H}_{\alpha}$ line for this star and although it is a relatively inactive star, they observed a day to day variation which they say is evidence for magnetic heating. Marcy \& Chen (1992) measured a vsini for G1 526 of $2.6 \pm 0.4 \mathrm{~km} \mathrm{~s}^{-1}$, which is large for an inactive dM1 star compared to the average measurements of Paper VII, but remains significantly smaller than the values for dM1e stars (Marcy \& Chen 1992; and Paper VII).

When comparing the observations and models of Houdebine $\&$ Stencel (1997), Gl 526 appears to be a relatively inactive star. Giampapa, Worden \& Linsky (1982) found a small plage filling 

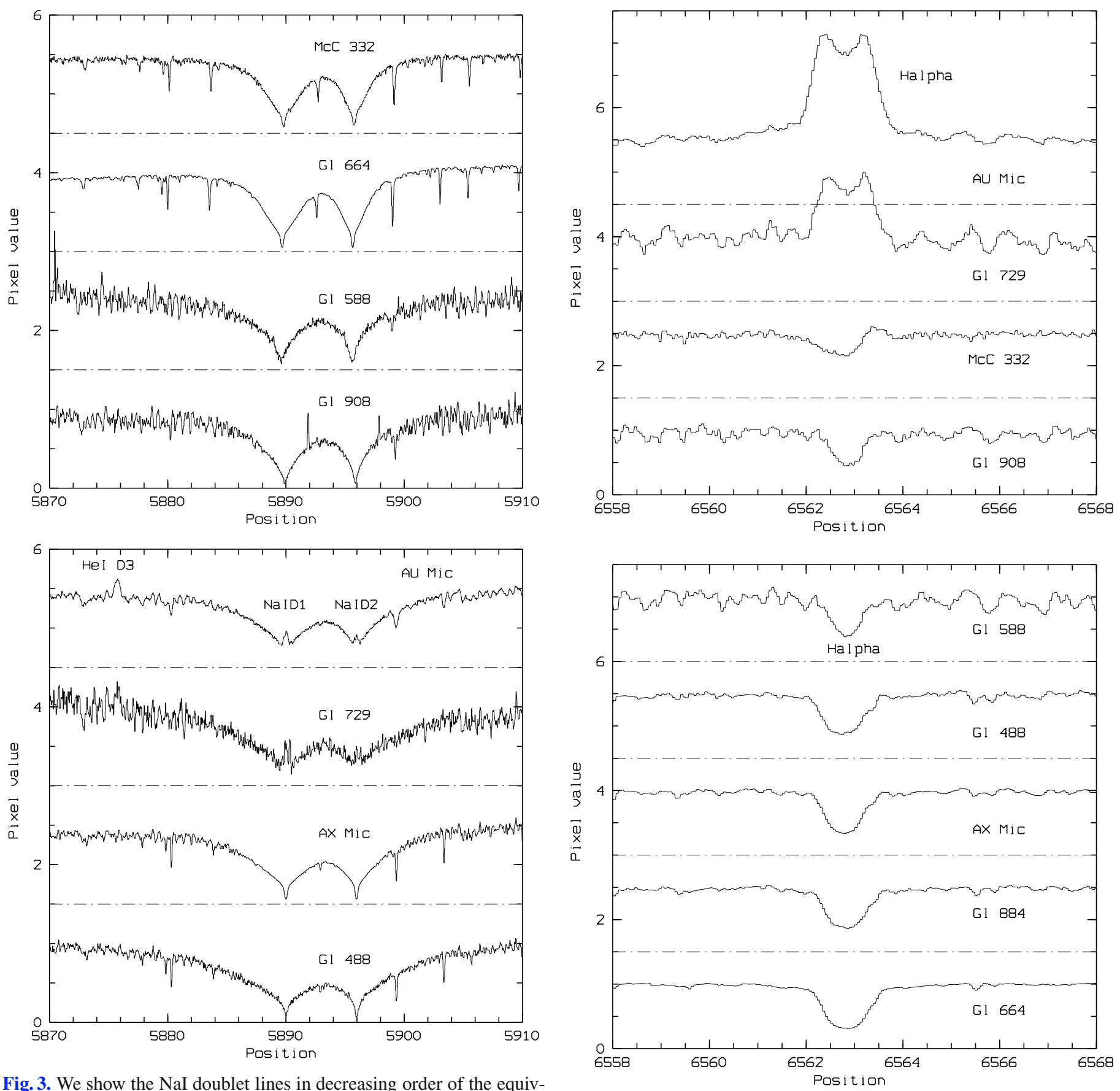

Fig. 3. We show the NaI doublet lines in decreasing order of the equivalent width. The HeI D3 line is also visible on the left for emission line stars.

factor of only $1.3 \%$. This star has also been studied in more detail by Houdebine (2009b). They produced a two-component model chromosphere for this star and found that this star has a rather low pressure chromosphere. Contrary to Giampapa et al. (1982), they found a plage filling factor of $36 \%$. In general, they found that the filling factor does not vary much with the activity level, but that it is the chromospheric pressure that diminish with the activity level.

\subsection{Gl 588}

Gl 588 is a dM4 star of v magnitude 10.1. For Gl 588, we observed the $\mathrm{H}_{\alpha}, \mathrm{H}_{\beta}, \mathrm{H}_{\epsilon}$, Ca II H, Ca I 6572 lines, the Ca II IR triplet lines, the $\mathrm{Na}$ I doublet and the $\mathrm{Pa}_{\epsilon}$ line.

Fig. 4. We show the $\mathrm{H}_{\alpha}$ line in decreasing order of the equivalent width. Note the interesting P-Cygni profile for McC 332.

The $\mathrm{H}_{\alpha} E W$ is rather large; $0.46 \pm 0.05 \AA$. This suggests that Gl 588 is an intermediate activity star, although its CaII line fluxes are relatively low; $-0.35 \pm 0.01 \AA$. More detailed modelling is required to make conclusions regarding the activity level of this star.

The NaI doublet line $E W$ is not large, compared to G1 729, a $\mathrm{dM} 4.5 \mathrm{e}$ star. This comes from the fact that the line width is much smaller in G1 588. Gl 588 has no detectable $\mathrm{H}_{\beta}$. It has also the weakest and narrowest CaII IR triplet lines (besides dMe stars) which also indicates a small metallicity for this star. These lines are unlikely to be filled-in because of the relatively weak activity level of G1 588, and because even Gl 729, a dM4.5e star does not show any core emission in these lines. Therefore, the weak 

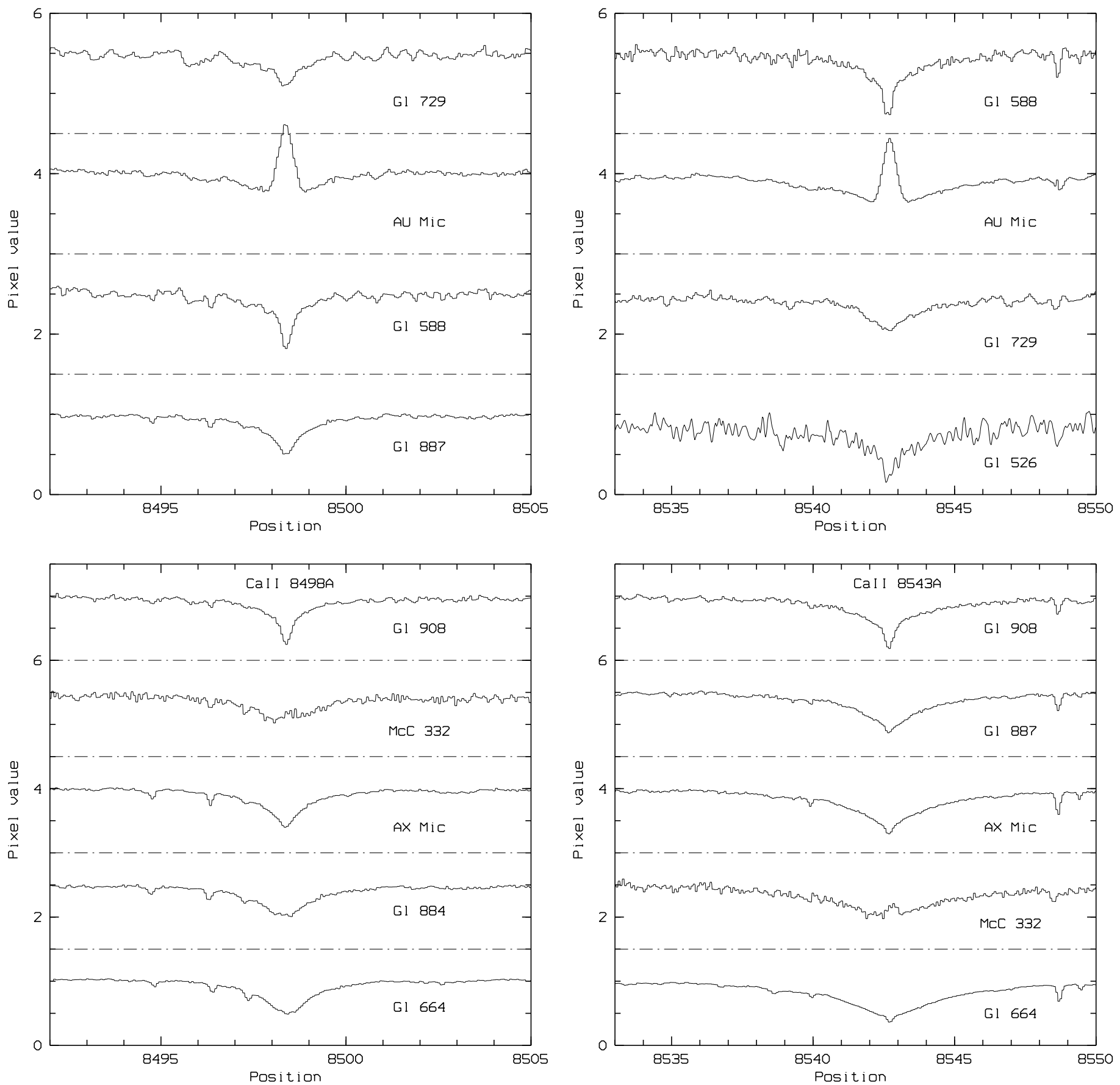

Fig. 5. We show the CaII IR triplet line at $8498 \AA$ in decreasing order of the equivalent width. Note the emission core in AU Mic. Gl 729 has no core emission although it is a dMe star.

Fig. 6. We show the CaII IR triplet line at $8542 \AA$ in decreasing order of the equivalent width. Note the emission core in AU Mic. Gl 729 has no core emission although it is a dMe star.

nature of the CaII IR triplet lines in the spectrum of Gl 588 is probably due to low metallicity. This is further emphasized by the fact that these lines are also narrower than for other stars, a signature of low metallicity. Such an effect of metallicity was also found for the NaI doublet lines in $\mathrm{dM} 1$ stars (Houdebine 2009d, Paper XIII). This would explain why the CaII resonance lines are weak whereas $\mathrm{H}_{\alpha}$ is not. $\mathrm{Pa}_{\epsilon}$ was also detected which is consistent with the fact that $\mathrm{H}_{\alpha}$ is relatively strong for a dM4 star.

Robinson et al. (1990) gave measurements of chromospheric radiative losses, of $\log F_{H}=3.89 \mathrm{ergs} \mathrm{s}^{-1} \mathrm{~cm}^{-2}$ and $\log F_{K}=$ $4.01 \mathrm{ergs} \mathrm{s}^{-1} \mathrm{~cm}^{-2}$ respectively for the $\mathrm{Ca}$ II $\mathrm{H}$ and $\mathrm{K}$ lines.

They also gave an equivalent width for $\mathrm{H}_{\alpha}$ of $0.45 \AA$, which is comparable to the value we report here; $0.455 \pm 0.05 \AA$. Mathioudakis \& Doyle (1991) also observed $\mathrm{H}_{\alpha}$ but at a lower resolution (1.4 $\AA$ ). They measured an $\mathrm{H}_{\alpha}$ equivalent width of only $0.32 \AA$. This is significantly smaller than other observations, and we believe this is due to the low resolution used. But this difference could also be due to a different choice in the level of the continuum which is difficult to determine for these stars. Mathioudakis \& Doyle (1991) also measured the flux received at Earth in the Ca II K line; $4.1 \times 10^{-14} \mathrm{erg} \mathrm{cm}^{-2} \mathrm{~s}^{-1}$, which yields a surface flux of $\log F_{\text {CaIIK }}=4.33 \mathrm{ergs} \mathrm{s}^{-1} \mathrm{~cm}^{-2}$. 


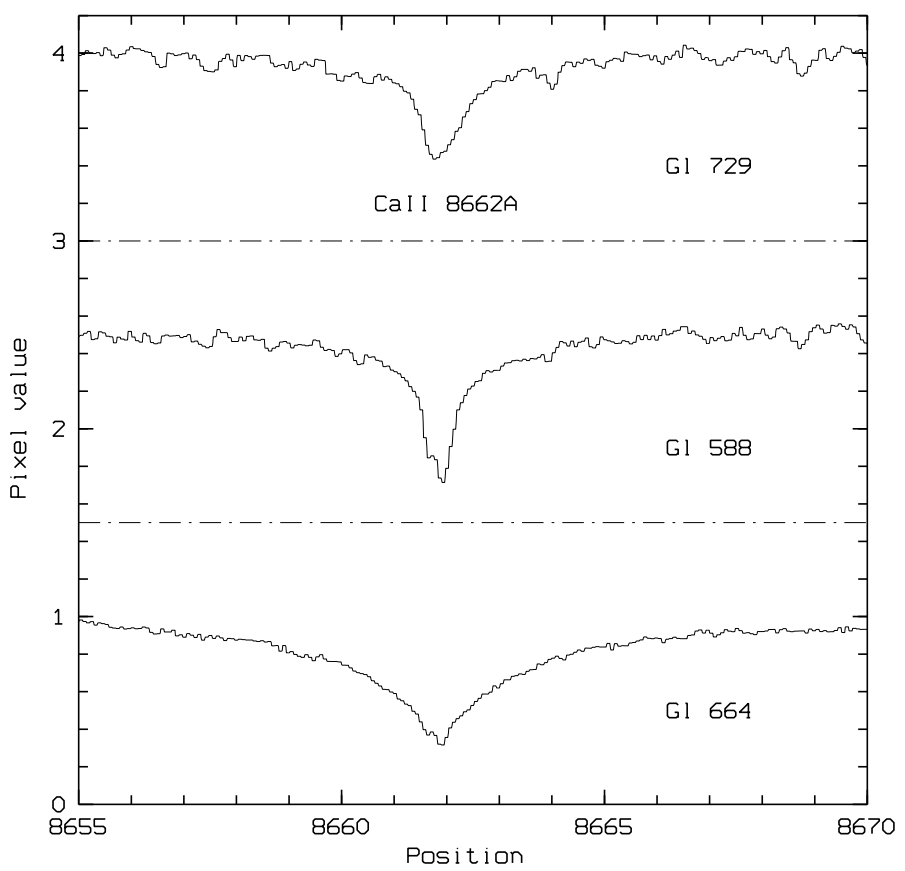

Fig. 7. We show the CaII IR triplet line at $8662 \AA$ in decreasing order of the equivalent width. Gl 729 has no core emission although it is a dMe star.

Mathioudakis \& Doyle (1992) give a surface flux in the Mg II lines of $\log F_{\mathrm{MgII}}=4.98 \mathrm{ergs} \mathrm{s}^{-1} \mathrm{~cm}^{-2}$ which is higher than the flux in the Ca II lines.

\subsection{Gl 664}

Gl 664 is a dK5 star. It belongs to the nearby triple system 36 Ophiuchi (Gl $664=36$ Ophiuchi C). An attempt to determine the orbits of A and B was performed by Brosche (1960). He found two possibilities characterized by $a=25.5^{\prime \prime}$ and $P=11547 \mathrm{yr}$, and $a=13.8^{\prime \prime}$ and $P=548.7$ yr. The second solution gives a mass of $0.73 M_{\odot}$ for each star in the system for an eccentricity of the orbit $\leq 0.90$ (Cayrel de Strobel et al. 1989). The distance of the $\mathrm{C}$ component to the $\mathrm{AB}$ components is much larger and its orbit cannot be determined. Cenarro et al. (2006) found that for this star the spectral type is dK5, $T_{\text {eff }}=4540 \mathrm{~K}$, $\log (g)=4.54$ and $[\mathrm{M} / \mathrm{H}]=-0.37$. Diaz et al. (2007) also give $[\mathrm{M} / \mathrm{H}]=-0.21$. This gives an estimate of the error on this measurement. It is therefore metal poor.

For Gl 664, we observed the $\mathrm{H}_{\alpha}, \mathrm{H}_{\beta}$, Ca I 6572 lines, the $\mathrm{Ca}$ I IR triplet lines, the $\mathrm{Na}$ I doublet and the $\mathrm{Pa}_{\epsilon}$ line. Cayrel de Strobel et al. (1989) also observed the Ca II 8498.06 and Ca II 8542.14 IR triplet lines at high resolution. They measured a central depth of 0.46 and 0.61 (respective to the adjacent continuum) respectively for the 8498.06 and the 8542.14 lines.

Early high resolution observations of $\mathrm{H}_{\alpha}$ by Kraft et al. (1964) gave a $F W H M$ for this line of $0.91 \AA$, which is close to the value we observe here of $1.04 \AA$. Robinson et al. (1990) observed Gl 664 at high resolution in the $\mathrm{H}_{\alpha}$ and $\mathrm{Ca}$ II $\mathrm{H}$ and $\mathrm{K}$ lines. They calculated a "chromospheric flux" (flux in the emission line core) in the $\mathrm{H}$ and $\mathrm{K}$ lines of respectively $\log F_{H}=5.16 \mathrm{erg} \mathrm{cm}^{-2} \mathrm{~s}^{-1}$ and $\log F_{K}=5.28 \mathrm{erg} \mathrm{cm}^{-2} \mathrm{~s}^{-1}$. They also measured an $\mathrm{H}_{\alpha}$ equivalent width of $750 \mathrm{~m} \AA$. Herbst \& Miller (1989) performed some $\mathrm{H}_{\alpha}$ photometry and measured an $\mathrm{H}_{\alpha}$ equivalent width of $850 \mathrm{m \AA}$. Here we report a significantly smaller equivalent width of $650 \pm 50 \mathrm{~m} \AA$.
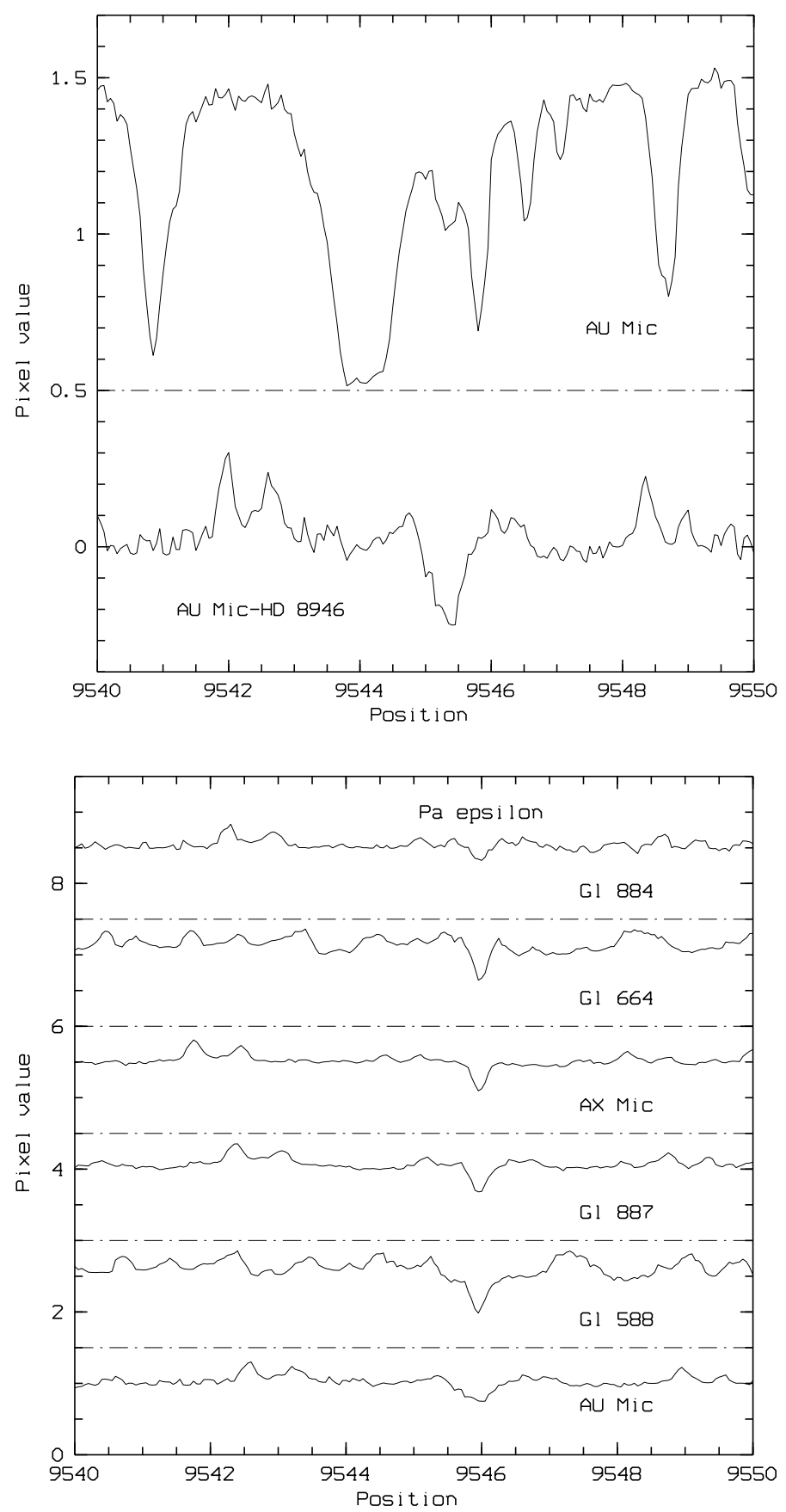

Fig. 8. We show here the $\mathrm{Pa}_{\epsilon}$ line at $9545 \AA$. In the upper panel, we show the normalized spectrum for AU Mic, together with the spectrum of AU Mic minus that of HD 8946 (an A0 star). In the lower panel, we show the spectra of our targets to which has been subtracted the spectrum of HD 8946, plus the constant 1 .

Baliunas et al. (1995) report on regular observations in the Ca II H and K lines (narrow band photometry) since 1966. They measured an average index $S$ of 0.770 and also found an activity cycle period of 21 years. Henry et al. (1996) observed more than 800 southern stars in the region of the $\mathrm{Ca}$ II $\mathrm{H}$ and $\mathrm{K}$ lines with a medium resolution spectrograph. They inferred an $S$ index of 1.07, somewhat larger than Baliunas et al.' (1995). Wright et al. (2004) report an $S$ index of 0.858 a bit higher than Baliunas et al.' (1995). Cincunegui et al. (2007) give a mean S index of 0.76 . The mean of all measures is $S=0.865$ which is relatively 


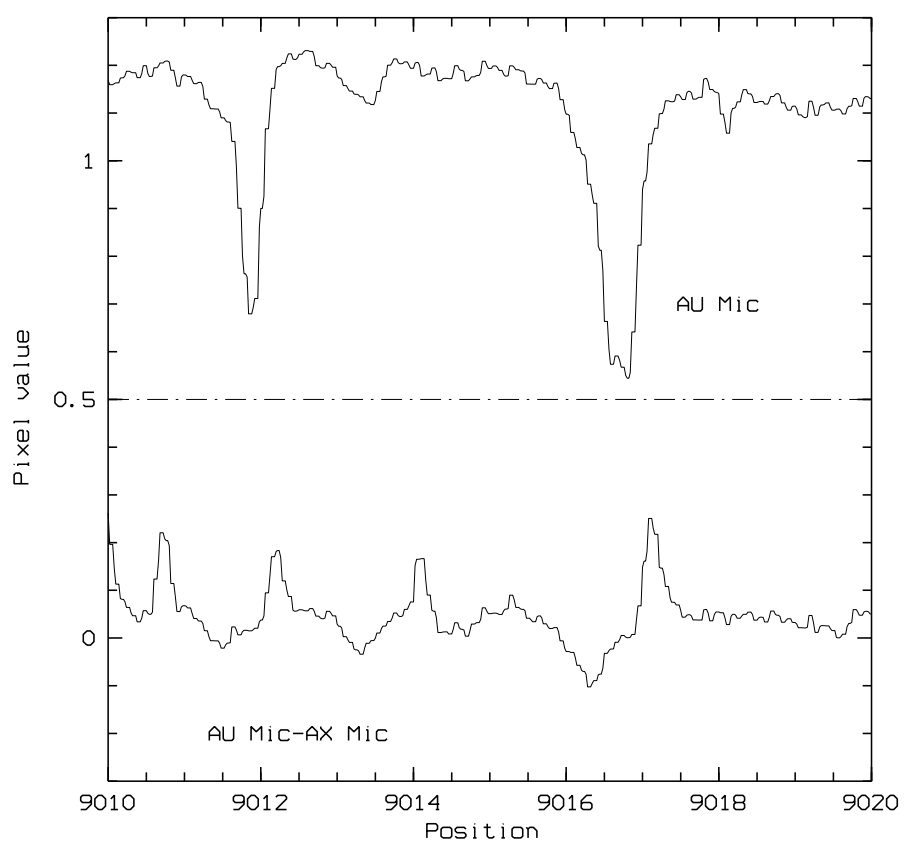

Fig. 9. We show the region of the $\mathrm{Pa}_{8}$ line at $8662 \AA$, for $\mathrm{AU}$ Mic and AU Mic-AX Mic. There seems to be no real detection of this line at $9015 \AA ̊$ in our spectra.

high (all these measures are in the Mount Wilson $S$ index). The variations, from 0.76 to 1.07 are due to the variability of the star (Baliunas et al. 1995).

The He I D3 line (5876 ̊) was observed at high resolution for Gl 664 by Saar et al. (1997). They observed an equivalent width for this line of $11 \pm 4 \mathrm{~m} \AA$. Although this value is weak, it is evidence for a chromosphere. Tripicchio et al. (1999) observed the $\mathrm{K}$ I $\lambda 7699$ resonance line at high resolution and measured an equivalent width of $460 \mathrm{m \AA}$. Gl 664 was also observed in the Mg II k line with IUE: Scoville \& Mena-Werth (1998) measured a FWHM for the Mg II k line of $0.39 \AA$.

Baliunas et al. (1983) observed a rotation period for this star of $18.0 \pm 0.5$ days. Saar and Osten (1997) investigated rotation and turbulence in a number of late type dwarfs and found for Gl 644 that $v \sin i$ and $v_{\text {mac }}$ (the macroturbulent velocity) are $0.3 \mathrm{~km} \mathrm{~s}^{-1}$ and $1.5 \mathrm{~km} \mathrm{~s}^{-1}$ respectively. Fekel (1997) also calculated these parameters and found values of $2.2 \mathrm{~km} \mathrm{~s}^{-1}$ and $2.0 \mathrm{~km} \mathrm{~s}^{-1}$ respectively. Saar \& Osten (1997) also inferred a rotation period of 18.5 days for this star, which makes it a relatively slow rotator. Gl 664 was also detected in X-rays, which is evidence of a hot corona, with an X-ray luminosity $L_{\mathrm{x}}=$ $5.6 \times 10^{27} \mathrm{erg} \mathrm{s}^{-1}$ (Hunsch et al. 1999).

$\mathrm{H}_{\alpha}$ is the strongest for gl 664 in our sample stars with an $E W$ of $640 \pm 50 \mathrm{~mA}$. Its CaII H \& K fluxes are relatively strong, indicating that it is an intermediate activity star. Its strong $\mathrm{H}_{\alpha}$ and $\mathrm{H}_{\beta}$ also reflects the fact that Balmer lines increase in absorption with decreasing spectral type. $\mathrm{Pa}_{\epsilon}$ was detected but is relatively weak with an $E W$ of $84 \pm 10 \mathrm{~m} \AA$. Unfortunately there are no grids of model chromospheres available for dK5 stars. It is therefore difficult to define its activity level. The comparison of these observations with the models of dM1 stars of Paper VI indicate that this is an intermediate activity star, with an $\mathrm{H}_{\alpha}$ absorption close to the maximum value for $\mathrm{dK} 5$ stars.

\subsection{Gl 729}

G1 729 is a dM4.5 $\mathrm{H}_{\alpha}$ emission line star. For G1 729, we observed the $\mathrm{H}_{\alpha}, \mathrm{H}_{\beta}$, Ca I 6572 lines, the Ca II IR triplet lines, the Na I doublet and the He I D3 line.

Young et al. (1989) report on $\mathrm{H}_{\alpha}$ observations of G1 729. They give an "excess equivalent width" of $1.72 \AA$ for Gl 729 . This "excess equivalent width" is defined as the measured equivalent width minus the equivalent width of the lower envelope of the graph given by Stauffer \& Hartmann (1986). However, according to calculations by Houdebine et al. (Papers III and VI), the maximum absorption in $\mathrm{H}_{\alpha}$ corresponds to intermediate activity levels which cannot be taken as a reference for minimum radiative losses. We recovered the original equivalent widths from the relation they used to calculate their "excess equivalent width". We find $\mathrm{H}_{\alpha}$ equivalent widths of $-2.14 \AA,-1.39 \AA$ and $-1.35 \AA$, which are of the same order as the value we measured; $-1.23 \AA$ A. Herbst \& Layden (1987) measured photometrically an $\mathrm{H}_{\alpha}$ equivalent width of $-1.11 \AA$, which is again close to our measurement.

Panagi \& Mathioudakis (1993) give a surface flux in the Ca II $\mathrm{H} \& \mathrm{~K}$ lines of $\log F=5.95 \mathrm{erg} \mathrm{cm}^{-2} \mathrm{~s}^{-1}$, which is high, and higher than for AU Mic which has a larger $\mathrm{H}_{\alpha}$ equivalent width. Rauscher \& Marcy (2006) measured $E W$ of the $\mathrm{H}$ and $\mathrm{K}$ lines of $-3.93 \AA$ and $-5.82 \AA$ respectively. Jenkins et al. (2006) found $\log R_{H K}^{\prime}=4.29$ only $\left(R_{H K}^{\prime}\right.$ is a measure of the chromospheric contribution to the $\mathrm{H} \& \mathrm{~K}$ lines, see Noyes et al. 1984). This is in significant disagreement with the Panagi \& Mathioudakis (1993) measurement. Landsman \& Simon (1993) measured a $\mathrm{Ly}_{\alpha}$ flux at Earth of $1.40 \times 10^{-12} \mathrm{erg} \mathrm{cm}^{-2} \mathrm{~s}^{-1}$, which taking into account the distance of the star yields a flux of $6.6 \times 10^{5} \mathrm{erg} \mathrm{cm}^{-2} \mathrm{~s}^{-1}$ on the stellar surface. Doyle et al. (1990) give a slightly higher surface flux of $8.5 \times 10^{5} \mathrm{erg} \mathrm{cm}^{-2} \mathrm{~s}^{-1}$, and also a Mg II h \& k flux of $4.0 \times 10^{5} \mathrm{erg} \mathrm{cm}^{-2} \mathrm{~s}^{-1}$.

Johns-Krull \& Valenti (1996) observed the Fe I $8468.40 \AA$ line. From their observations they inferred that $50 \%$ of G1 729 is covered with magnetic fields of $2.6 \pm 0.3 \mathrm{kG}$ strength.

\section{6. $A U$ Mic ( $g l 803)$}

AU Mic is one of the best studied active red dwarfs, it is also a flare star (e.g. Kunkel 1973). It is known to have a disk of debris (e.g. Fitzgerald et al. 2007). For AU Mic, we gathered one of the most complete set of observations: the $\mathrm{H}_{\alpha}, \mathrm{H}_{\beta}, \mathrm{H}_{\epsilon}, \mathrm{Ca} I I \mathrm{H}$, Ca I 6572 lines, the Ca II IR $8498 \AA$ and $8542 \AA$ triplet lines, the $\mathrm{Na}$ I doublet, the $\mathrm{He} \mathrm{I} \mathrm{D} 3$, the $\mathrm{Pa}_{\epsilon}$ and $\mathrm{Pa}_{8}$ lines.

A number of studies have been performed on its Ultra Violet spectrum. For instance Butler et al. (1981), Linsky et al. (1982), Ayres et al. (1983), Oranje (1986), Butler et al. (1987), Doyle (1987), Byrne \& Doyle (1989, 1990), Doyle et al. (1990), Landsman \& Simon (1993) and Quin et al. (1993) have investigated IUE observations. More recently, Maran et al. (1994), Linsky \& Wood (1994), Wood et al. (1997), Redfield \& Linsky (2002) studied HST/GHRS spectra of AU Mic. Pagano et al. (2000) have investigated HST/STIS spectra. Del Zanna et al. (2002) analyzed FUSE observations while Schrijver et al. (1995), Monsignori Fossi \& Landini (1994), and Monsignori Fossi et al. (1996), report on observations at shorter wavelengths with EUVE. Most of these studies report spectral line fluxes of UV lines and related empirical correlations. Linsky \& Wood (1994) found that C IV and Si IV line profiles show two components with respective widths of about $29 \mathrm{~km} \mathrm{~s}^{-1}$ and $170 \mathrm{~km} \mathrm{~s}^{-1}$. Wood et al. (1997) interpret this line broadening in terms of 

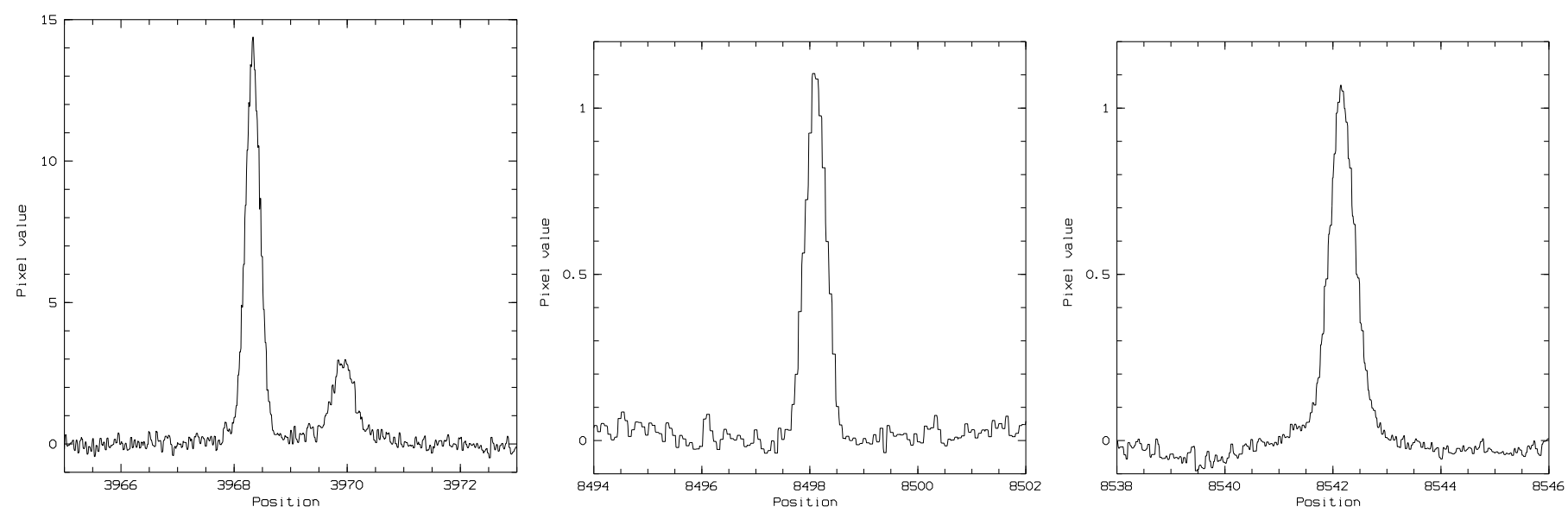

Fig. 10. We show here the difference spectra AU Mic-Gl 887. Note the wings for the $8542 \AA$ line.
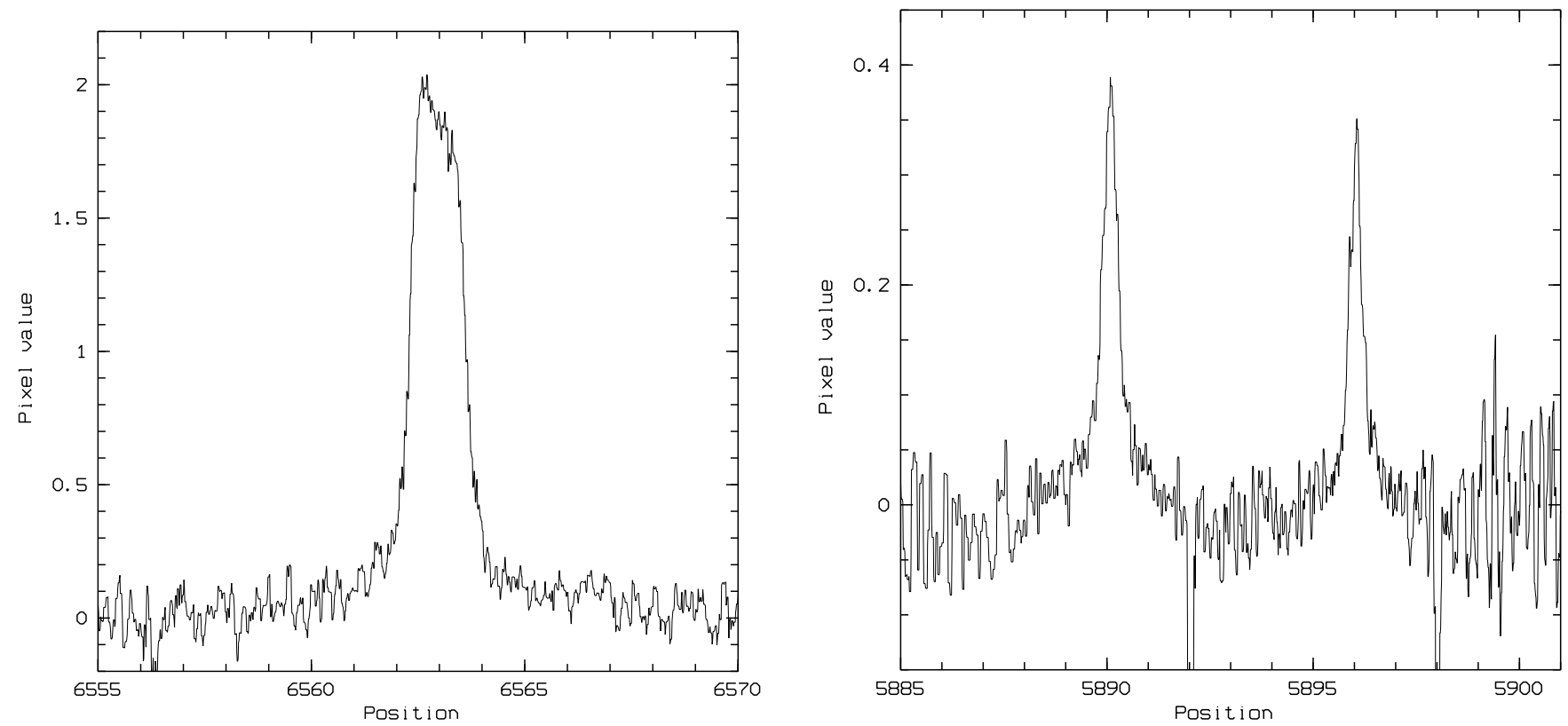

Fig. 11. We show here the difference spectra AU Mic-Gl 908.

possible heating mechanisms. Pagano et al. (2000) also report on two-Gaussian fits to the line profiles. Emission measure distributions were derived notably by Butler et al. (1987), Quin et al. (1993), Maran et al. (1994), Schrijver et al. (1995), Monsignori Fossi et al. (1996), Pagano et al. (2000). Del Zanna et al. (2002) derived a rather complete and interesting emission measure distribution from $10^{4} \mathrm{~K}$ to $2 \times 10^{7} \mathrm{~K}$ from combined observations of FUSE, STIS and EUVE spectra.

Robinson et al. (1990) measured chromospheric fluxes in the CaII H and K lines of $4.99 \times 10^{5} \mathrm{erg} \mathrm{cm}^{-2} \mathrm{~s}^{-1}$ and $5.66 \times$ $10^{5} \mathrm{erg} \mathrm{cm}^{-2} \mathrm{~s}^{-1}$ respectively. Mathioudakis \& Doyle (1989) measured an MgII surface flux of $\log _{\mathrm{MgII}}=6.06 \mathrm{erg} \mathrm{cm}^{-2} \mathrm{~s}^{-1}$. For $\mathrm{H}_{\alpha}$, we measured an $E W$ of $-2.47 \pm 0.1 \AA$. Robinson et al. (1990) measured an equivalent width of $-2.01 \AA$. Herbst \& Layden (1987) derived photometrically an $\mathrm{H}_{\alpha}$ equivalent width of $-1.90 \AA$. Worden et al. (1981) also obtained high resolution observations of the Balmer lines. They measured equivalent widths of $-8.70 \AA$ and $-1.56 \AA$ for $\mathrm{H}_{\alpha}$. The former value, which is very high, probably corresponds to a flare. Similarly, for $\mathrm{H}_{\beta}$ they measured a high value of $-3.68 \AA$, which is much larger than our value of $-1.9 \AA$. Pettersen (1989) measured $\mathrm{H}_{\alpha}$ equivalent widths of $-2.01 \AA$ and $-2.08 \AA$. Doyle et al. (1997) inferred a $\mathrm{Ly}_{\alpha}$ flux of $1.1 \times 10^{-11} \mathrm{erg} \mathrm{cm}^{-2} \mathrm{~s}^{-1}$ at Earth, which yield a $\mathrm{Ly}_{\alpha}$ to $\mathrm{H}_{\alpha}$ ratio of 3.3. Short \& Doyle (1998) report on high resolution observations of the $\mathrm{Pa}_{\beta}$ line and proposed semiempirical model atmospheres to reproduce their observations. This line appears to be in absorption which gives interesting constraints on model atmospheres, especially about the lower chromosphere where the Paschen lines are formed (Short \& Doyle 1998, Papers II and III). Worden et al. (1981) also observed the NaI doublet for which they detected a core emission.

Pettersen (1989) has observed a number of chromospheric lines of interest for $\mathrm{AU}$ Mic. For the $\mathrm{Na} \mathrm{I}_{1}$ and $\mathrm{Na} \mathrm{I}_{2}$ line core emissions he gives an equivalent width of $-0.05 \AA$ and $-0.10 \AA$ and $F W H M$ of $0.22 \AA$ and $0.40 \AA$ respectively. Here, we measured line core equivalent widths of $-0.063 \pm 0.01 \AA$ and $-0.032 \pm 0.01 \AA$ for the D1 and D2 lines respectively. Pettersen (1989) also obtained unique observations of the Ca II infrared triplet: he found these lines to have an emission core with equivalent widths of $-0.42 \AA,-0.55 \AA$ and $-0.37 \AA$ respectively for the $8498 \AA, 8542 \AA$ and $8662 \AA$ lines. However, some of these observations have a rather low signal to noise and the spectral 
resolution was typically $0.46 \AA$ at $\mathrm{H}_{\alpha}$, giving a resolving power of only 15000 . Our observations have a higher resolving power and better signal to noise ratio. For the Ca II $8498 \AA$ and $8542 \AA$ line cores, we measured equivalent widths of $-0.38 \pm 0.05 \AA$, $-0.45 \pm 0.05 \AA$ respectively. These values are a bit weaker than those of Pettersen (1989).

Giampapa et al. (1982) derived a 0.54 and 0.42 plage filling factor based on CaII and MgII observations respectively, whereas in Paper II we derived a 0.30 filling factor based on the modelling of the Balmer and Lyman lines. More recently, in Paper XII, we derived a plage filling factor of only $9 \%$ from a two-component model chromosphere. These values represent a large scatter: unfortunately, at this stage it is difficult to reach a conclusion. In Paper XII we find that a new grid of model chromospheres with lower temperature minima would be preferable. But the twocomponent chromospheric modelling seems the most accurate approach, particularly since the quiescent region contribution is important (Paper XII).

Similarly to Pettersen (1989) we observe a core emission in the NaI doublet lines as well as in the CaII IR triplet lines. This core emission in the CaII IR triplet is pretty strong. The $\mathrm{Pa}_{\epsilon}$ line is detected (Fig. 8). It's profile is interesting: there seems to be wing emission and a core absorption. This profile is broader than for other stars, probably because of rotation. This spectral line is of great interest for chromospheric modelling because it is formed at a lower temperature than the Balmer lines (Short \& Doyle 1998a; Papers II and III).

We also observed the $\mathrm{Pa}_{8}$ line for AU Mic. Unfortunately we did not observe a reference star for this line, but we observed it also for AX Mic. If we compute the difference of these two spectra AU Mic-AX Mic, the $\mathrm{Pa}_{8}$ line should appear as a P-Cygni profile: an emission component from AX Mic and an absorption component for AU Mic, because they do not have the same radial velocity. The reason for calculating such difference spectra is to remove the telluric lines which are strong at this wavelength (Fig. 9 for $\mathrm{Pa}_{8}$ and Fig. 8 for $\mathrm{Pa}_{\epsilon}$ ). In the case of $\mathrm{AU}$ Mic-AX Mic, all photospheric line profiles appear as P-Cygni profiles because these two stars have close spectral types.

We computed the difference spectrum; AU Mic-AX Mic. We show the difference spectrum in Fig. 9. We can note a number of spectral features, but none at the expected wavelength of the $\mathrm{Pa}_{8}$ line $(9015 \AA)$. Therefore this line is not detected either for AU Mic nor AX Mic.

Is G1 729 more active than AU Mic? Our observations show that there are interesting and important differences between the two stars. A weak core emission in the NaI doublet lines is detected for both stars (Fig. 3). But, contrary to AU Mic, there is no core emission in the CaII IR triplet lines for Gl 729 (Figs. 5 to 7). In addition $\mathrm{H}_{\alpha}$ is much stronger in AU Mic than in G1 729, but the surface fluxes in the $\mathrm{Ca}$ II resonance lines are larger for Gl 729 than for AU Mic. At this stage we cannot explain the different properties of the chromosphere for these two stars, all we can say is that there are important differences. The detailed modelling of these lines will have to explain what these differences are due to.

\section{7. $A X \operatorname{Mic}(\mathrm{Gl} 825)$}

AX Mic is a bright $(v=6.64) \mathrm{dK} 7$ star according to its molecular bandstrengths (Hawley et al. 1996). This star has often been classified as a dM1 star (e.g. Byrne \& Doyle 1990; Jenkins et al. 2006), but its infrared color $(R-I)_{\mathrm{c}}=0.865$ is too small for a dM1 star (typically 1.2, Paper VI). Gershberg et al. (1999) classify this star as a flare star. For AX Mic, we observed the $\mathrm{H}_{\alpha}, \mathrm{H}_{\beta}, \mathrm{H}_{\epsilon}, \mathrm{Ca}$ II H, Ca I 6572 lines, the Ca II IR $8498 \AA$ and $8542 \AA$ triplet lines, the $\mathrm{Na}$ I doublet and the $\mathrm{Pa}_{\epsilon}$ lines.

Our observations of $\mathrm{H}_{\alpha}$ show that this star has a large $E W$ of $0.592 \pm 0.05 \AA$ (Robinson et al. 1990 , give $0.60 \AA$ ). This $E W$ is partly due to the relatively early spectral type of AX Mic. Our observations of the CaII $\mathrm{H}$ line shows that this line is weak compared to most of the equivalent widths observed for intermediate activity dM1 stars (Paper XI); the $E W$ of this line is only $-0.599 \AA$ A. Similarly, Robinson et al. (1990) give a surface flux for the CaII $\mathrm{H}$ line of only $\log F_{H}=4.52 \mathrm{erg} / \mathrm{s} / \mathrm{cm}^{2}$ and Panagi $\&$ Mathioudakis (1993) give $\log F_{H}=5.05 \mathrm{erg} / \mathrm{s} / \mathrm{cm}^{2}$. Jenkins et al. (2006) found a similar value of $\log F_{H}=4.93 \mathrm{erg} / \mathrm{s} / \mathrm{cm}^{2}$. Only the detailed NLTE modelling can explain why we have a strong $\mathrm{H}_{\alpha}$ and weak CaII fluxes. But the early comparison with the models for the near spectral type dM1 stars (Papers XI and XII) shows that this is a relatively low activity star, and its large $\mathrm{H}_{\alpha}$ equivalent width is at least partly due to its earlier spectral type.

The CaII $\mathrm{H}$ line has an interesting profile; it shows a central absorption indicating a relatively high chromospheric opacity. This is in agreement with the fact that stars with large radii are slightly metal rich (Houdebine 2008, Paper VII). This can also be due to the fact that in $\mathrm{dK}$ stars the chromospheric temperature gradient is smaller than in dM stars, simply because the temperature minimum is higher. This smaller temperature gradient yields a higher mass loading in the region of formation of the CaII lines, and therefore a higher optical depth for a given column mass of the transition region. Calculations by Houdebine (1994) clearly show that the relative intensity of the central absorption in the CaII lines increases with increasing effective temperature for a given transition region column mass.

The $\log F_{\mathrm{MgII}}$ of 5.18 given by Mathioudakis \& Doyle (1989) (Panagi \& Mathioudakis 1993, give 5.29) is typically that of a dK7 star. Byrne \& Doyle (1989) give the surface fluxes in some UV lines for AX Mic, but these values are somewhat erroneous because they use a stellar radius of only $0.58 R_{\odot}$. Hunsch et al. (1999) found a very small X-ray luminosity of $1.1 \times 10^{27} \mathrm{erg} / \mathrm{s}$. This is in agreement with the small CaII $\mathrm{H}$ flux.

We detected $\mathrm{H}_{\beta}$ and $\mathrm{Pa}_{\epsilon}$ in absorption in $\mathrm{AX}$ Mic. On the other hand, $\mathrm{Pa}_{8}$ was not detected (Fig. 9).

\subsection{Gl 884}

G1 884 was often classified as a dM1 star (e.g. Robinson et al. 1990), however, its infrared color $(R-I)_{\mathrm{c}}=0.770$ (Robinson et al. 1990) is not compatible with this spectral type (Paper VI). In The CDS database we find a spectral type of dK5 which is in better agreement. More recently, Gray et al. (2006) studied this star and found a spectral type of dK7. For Gl 884, we observed the $\mathrm{H}_{\alpha}, \mathrm{H}_{\epsilon}, \mathrm{Ca}$ II H, Ca I 6572 lines, the Ca II IR $8498 \AA$ triplet line and the $\mathrm{Pa}_{\epsilon}$ line.

Robinson et al. (1990) found a surface flux in the CaII H \& $\mathrm{K}$ lines of respectively $\log F_{H}=4.88 \mathrm{erg} / \mathrm{s} / \mathrm{cm}^{2}$ and $\log F_{K}=$ $4.93 \mathrm{erg} / \mathrm{s} / \mathrm{cm}^{2}$, and an $E W$ of the CaII K line of $-1.32 \AA$. Here, we find an $E W$ of the CaII $H$ line of $-0.97 \pm 0.05 \AA$. Rauscher $\&$ Marcy (2006) found mean $E W$ of $-0.79 \AA$ and $-1.14 \AA$ respectively for the $\mathrm{H}$ and $\mathrm{K}$ lines. Gray et al. (2006) list a CaII $\mathrm{H} \& \mathrm{~K}$ flux of $\log F_{\text {CaII }}=4.643$. Wright et al. (2004) observed a CaII S index of $1.569 . \mathrm{H}_{\alpha}$ is relatively strong with an $E W$ of $0.605 \pm 0.05 \AA$. Herbst \& Layden (1987) found an $E W$ of $0.53 \AA$ and Panagi \& Mathioudakis (1993) quote an $E W$ of $0.60 \AA$ in better agreement with our measurement. 
Although the CaII line surface flux is relatively low, this is one of the most active stars in our sample, according to its CaII $\mathrm{H}$ line $E W$ (Fig. 1). Similarly to AX Mic, the CaII H line shows a weak central absorption. $\mathrm{H}_{\epsilon}$ is just about detected in weak emission although $\mathrm{H}_{\alpha}$ is in absorption. The CaII IR $8498 \AA$ line is rather broad and it seems there is a "filling-in" of the line core when compared to the spectrum of AX Mic for instance (which is deeper). This would be consistent with the much larger $\mathrm{CaII} \mathrm{H}$ $E W$ for Gl 884. This could be an interesting constraint for modelling. It is interesting to note that there is a possible weak filling in for this line for the two dK stars, Gl 664 and Gl 884, whereas we do not observe such filling in for the supposedly more active dM4.5e star Gl 729 .

The $\mathrm{Pa}_{\epsilon}$ line was detected in absorption and is the weakest in our sample, with only $46 \pm 10 \mathrm{~m} \AA$, that is almost four times weaker than in AU Mic.

\subsection{Gl 887}

Robinson et al. (1990) gave an infrared color of $(R-I)_{\mathrm{c}}=1.04$ for G1 887. This is very close to the infrared color of AU Mic $(R-I)_{\mathrm{c}}=1.10$ (Paper VII). It is in fact preferable to compare the $R-I$ infrared colors which are a good indicator of the effective temperature rather than the spectral type which varies for different classifications for M dwarfs (Paper VI). Gl 887 was classified as an M0.5 star (SIMBAD-CDS). It has therefore also a spectral type close to that of AU Mic. Hence, we can compare their spectra and look for the real contributions of plages in $\mathrm{AU}$ Mic. For Gl 887, we observed the $\mathrm{H}_{\epsilon}$, Ca II H lines, the Ca II IR $8498 \AA$ and $8542 \AA$ triplet lines and the $\mathrm{Pa}_{\epsilon}$ line.

Lopez-Morales (2007) gave $R=0.491 \pm 0.014 R_{\odot}$ (see also Segransan et al. 2003) and $[\mathrm{M} / \mathrm{H}]=-0.22 \pm 0.09 \mathrm{dex}$ (see also Bonfils et al. 2005). This can be compared to the values found in Paper VII from the radius/metallicity correlation; $R=0.579 R_{\odot}$ and $[\mathrm{M} / \mathrm{H}]=-0.092$ dex respectively. The values of radii inferred in Paper VII agree well in general with interferometric measurements. The difference in radii between the measurements of Lopez-Morales (2007) and ours is one of the largest in our sample in Paper VII. At this stage we have no explanation for this relatively important difference (error in parallax, in $R-I$ or in interferometry?). Additional measures will be necessary to reach a conclusion. The metallicity we derived in Paper VII for Gl 887 directly depends on its radius. This is the reason why there is also some difference between the two metallicity measurements. Therefore, Gl 887 has a small radius (compared to solar metallicity M1 dwarfs, Paper VII) and is a metal-poor dM1 star.

Gl 887 has a rather weak CaII H line $E W(-0.796 \pm 0.05 \AA)$ compared to AU Mic $(-4.55 \pm 0.2 \AA) . \mathrm{H}_{\epsilon}$ also seem to have a weak emission in Gl $887(-0.11 \pm 0.03 \AA)$, whereas $\mathrm{H}_{\alpha}$ is in absorption $(E W=0.45 \AA$, Panagi \& Mathioudakis 1993). Rauscher \& Marcy (2006) give equivalent widths of $-0.77 \AA$ and $-0.54 \AA$ respectively for the $\mathrm{H}$ and $\mathrm{K}$ lines, from several measurements. This is close to the value we observe. Robinson et al. (1990) give CaII H \& K fluxes of $\log F_{H}=4.68 \mathrm{erg} / \mathrm{s} / \mathrm{cm}^{2}$ and $\log F_{K}=4.62 \mathrm{erg} / \mathrm{s} / \mathrm{cm}^{2}$ respectively. Tinney et al. (2002) gave $\log R_{H K}^{\prime}=5.01 \mathrm{erg} / \mathrm{s} / \mathrm{cm}^{2}$ (see also Jenkins et al. 2006). Doyle et al. (1990) found $\mathrm{Ly}_{\alpha}$ and MgII surface fluxes of $5.32 \mathrm{erg} / \mathrm{s} / \mathrm{cm}^{2}$ and $5.23 \mathrm{erg} / \mathrm{s} / \mathrm{cm}^{2}$ respectively. The MgII flux is substantially larger than the CaII flux.

According to the CaII and $\mathrm{H}_{\alpha}$ equivalent widths, and to the models of Houdebine \& Stempels (1987, Paper VI), Gl 887 is a low activity dM1 star. This agrees with its low coronal luminosity; $L_{\mathrm{x}}=7 \times 10^{26} \mathrm{erg} / \mathrm{s}$, and with the fact that Gl 887 is slightly metal poor dM1 star (Paper VII).

We subtracted the spectra of G1 887 from those of AU Mic. We show the difference spectra in Fig. 10. The CaII H line does not show any central absorption, in spite of the fact, that for dM1e stars the chromosphere is optically thick in this line (Paper VI). There are interesting differences between the CaII IR triplet lines at $8498 \AA$ and $8542 \AA$. The latter line is a bit broader and shows some weak wings while the $8498 \AA$ line does not. These are interesting constraints for modelling.

\subsection{Gl 908}

Herbst \& Miller (1989) gave an infrared color $(R-I)_{K}=0.87$ (i.e. $\left.(R-I)_{C}=1.124\right)$. Therefore, this is a dM1 star that suits the selection criterion of Paper VI. It has the same spectral type as AU Mic and G1 887. In Paper VI, we found that for G1908: $R=0.498 R_{\odot}$ and $[\mathrm{M} / \mathrm{H}]=-0.271$. Bonfils et al. (2005) give an even lower metallicity of $[\mathrm{M} / \mathrm{H}]=-0.52$. Therefore, Gl 908 is a small radius (compared to solar metallicity M1 dwarfs, Paper VII) and metal poor M1 dwarf, that probably has a very low activity level (Paper VI). For Gl 908, we observed the $\mathrm{H}_{\alpha}$, $\mathrm{H}_{\epsilon}$, Ca II H, Ca I 6572 lines, the Ca II IR $8498 \AA$ and $8542 \AA$ triplet lines and the $\mathrm{Na}$ I doublet.

Stauffer \& Hartmann (1986) found an $\mathrm{H}_{\alpha} E W$ of $0.40 \AA$. Herbst \& Miller (1989) found $0.44 \AA$, Herbst \& Layden (1987) found $0.36 \AA$, and here we also find $0.36 \pm 0.05 \AA$. This is a weak $\mathrm{H}_{\alpha} E W$ according to Paper VI. This agrees with the small CaII $\mathrm{H} E W$ of only $-0.188 \pm 0.03 \AA$ that we observe. The average CaII H \& K equivalent width from various authors is $-0.245 \AA$ (Paper VI). The average of the two lines is a better diagnostic of activity because the line ratios vary significantly with activity level (Paper VI). This is a low metallicity, very low activity dM1 star (Papers VI and VII). The surface flux in the CaII $\mathrm{H} \& \mathrm{~K}$ lines is $\log F=4.88 \mathrm{erg} / \mathrm{s} / \mathrm{cm}^{2}$. Byrne (1993) gave an even lower value of $\log F=4.56 \mathrm{erg} / \mathrm{s} / \mathrm{cm}^{2}$. $L_{\mathrm{X}}$ given by Hunsch et al. (1999) is $1.7 \times 10^{27} \mathrm{erg} / \mathrm{s}$. This is also in agreement with its low vsini $\left(0.9 \mathrm{~km} \mathrm{~s}^{-1}\right.$, Paper VII) and long rotation period $P / \sin i=28 \mathrm{~d}$.

We can therefore compare the $\mathrm{H}_{\alpha}$ and NaI D1/D2 spectra of AU Mic and Gl 908. We cross-correlated the spectra of AU Mic and Gl 908 for a region where narrow photospheric features are present (not much affected by metallicity) in order to determine the wavelength shift. We shifted the spectrum of AU Mic to the reference frame of Gl 908 . We show the difference spectra in Fig. 11. The $\mathrm{H}_{\alpha}$ profile no longer shows a self-reversal and is clearly asymmetric in the line centre. We can also note some wing emission. In Paper II, we showed that the central absorption of the $\mathrm{H}_{\alpha}$ line is the main constraint on the models. A weaker absorption implies a higher transition region pressure and a higher chromospheric gradient. Here we did not take into account a filling factor for the quiescent regions, which means that in reality the central absorption is a bit larger than in Fig. 11. However, we conclude that our model given in Paper II is still a lower limit of the true plage model of AU Mic.

Figure 11 also shows that the NaI D1/D2 lines have inner wing emission as well as the core emission. In fact, most of the energy is contained in these wings. Again, these are interesting constraints for the modelling of dMe chromospheres. The narrow absorption features visible on the red wings of the $\mathrm{NaI}$ D1/D2 lines come from the low pressure sodium lamps of the nearby city of La Serena visible in the Gl 908 spectrum (Fig. 3). 


\subsection{McC 332 (HIP 104383)}

McC 332 is one of the most interesting stars that we observed. It was reported as a binary by Poveda et al. (1994). Later, speckle interferometry was reported by Balega et al. (2004). The primary has been classified as a dM2 star with $v=9.44$, while the secondary is a faint $\mathrm{M}$ dwarf with $v=13.4$. The primary has $(R-I)_{\mathrm{c}}=0.68$ and the secondary has $(R-I)_{\mathrm{c}}=1.42$ (Weis 1993). The primary's color is too blue for an M2 star, it is more probably a dK7 star (Bessel 1990). The color of the secondary indicates that it is a dM3.5e star (Bessel 1990). Gershberg et al. (1999) reported McC 332 as a flare star. It is most probably the fainter active companion that exhibits flares, because as shown from the spectra below it is much more active than the primary $\left(\mathrm{H}_{\alpha}\right.$ emission, stronger $\mathrm{Ca}$ II H emission). For $\mathrm{McC} 332$ we observed the $\mathrm{H}_{\alpha}, \mathrm{H}_{\beta}, \mathrm{H}_{\epsilon}$, Ca II H, Ca I 6572 lines, the Ca II IR $8498 \AA$ and $8542 \AA$ triplet lines and the Na I doublet.

McC 332 has been rarely observed. Wilson and Wooley (1970) reported a CaII H \& $\mathrm{K}$ index of +3 , which means a relatively strong emission. Herbst \& Layden (1987) observed an $\mathrm{H}_{\alpha}$ $E W$ of $0.63 \AA$ photometrically. Here we report a much weaker $\mathrm{H}_{\alpha} E W$, of only $0.29 \AA$. Our $\mathrm{H}_{\alpha}$ line profile is of P-Cygni type (Fig. 4) and our CaII H profile clearly shows a blue asymmetry (Fig. 14)! This is the first time that this star has been observed spectroscopically. Herbst \& Layden (1987) probably only observed the primary, whereas here we have spectra of both the primary and the secondary.

We extracted the 6565-6590 $\AA$ region of our $\mathrm{H}_{\alpha}$ spectra of $\mathrm{McC} 332$ and Gl 488. This region is interesting for crosscorrelations because it includes strong and unblended absorption features. We cross-correlated Gl 488 with itself and McC 332 with $\mathrm{Gl}$ 488. The resulting normalized cross-correlation profiles are shown in Fig. 12. We chose Gl 488 instead of Gl 908 which has a low vsini (Houdebine 2008) because the spectrum has a higher $\mathrm{S} / \mathrm{N}$ ratio.

The cross-correlation profile of Gl 488 has a $F W H M$ of $0.347 \AA$. This is $0.19 \AA$ larger than the expected instrumental $F W H M$ for a resolution of 40000 . This yields a possible vsini of $8.2 \mathrm{~km} \mathrm{~s}^{-1}$ for Gl 488 which seems too high for an inactive dK7 star (typically less than $3 \mathrm{~km} \mathrm{~s}^{-1}$ see Paper VII). Therefore, either the resolution was lower, or the width is due to the intrinsic width of the line profiles. All we can say is that the McC 332/Gl 488 cross-correlation profile is broader with a FWHM of $0.394 \AA$. This means that vsini for McC 332 is about $8.5 \mathrm{~km} \mathrm{~s}^{-1}$ larger than vsini for $\mathrm{Gl}$ 488. This is quite large for an $\mathrm{H}_{\alpha}$ absorption line star (Paper VII). This excess broadening is probably partly due to the fainter secondary Gaussian component discussed below.

We can note in Fig. 12 that the McC 332/Gl 488 crosscorrelation profile is asymmetric and shows a bump in its red wing. We fitted two Gaussians and found that the second fainter Gaussian is red shifted by $0.36 \AA$ with respect to the main Gaussian. We believe this is evidence that McC 332 is a spectroscopic binary with a dK7 principal component and a secondary component of later spectral type. The wavelength shift yields a velocity of $16 \mathrm{~km} \mathrm{~s}^{-1}$ only. The parameters of our two-Gaussian fit are summarized in Table 2.

We subtracted the $\mathrm{Gl} 488 \mathrm{H}_{\alpha}$ spectrum from the MCC 332 $\mathrm{H}_{\alpha}$ spectrum. The difference spectrum is shown in Fig. 13. A nice emission $\mathrm{H}_{\alpha}$ profile appears with a possible self-reversal. This emission profile is red-shifted by about $0.20 \AA$ with respect to the $\mathrm{H}_{\alpha}$ absorption profile of $\mathrm{Gl} 488$. This further strengthens

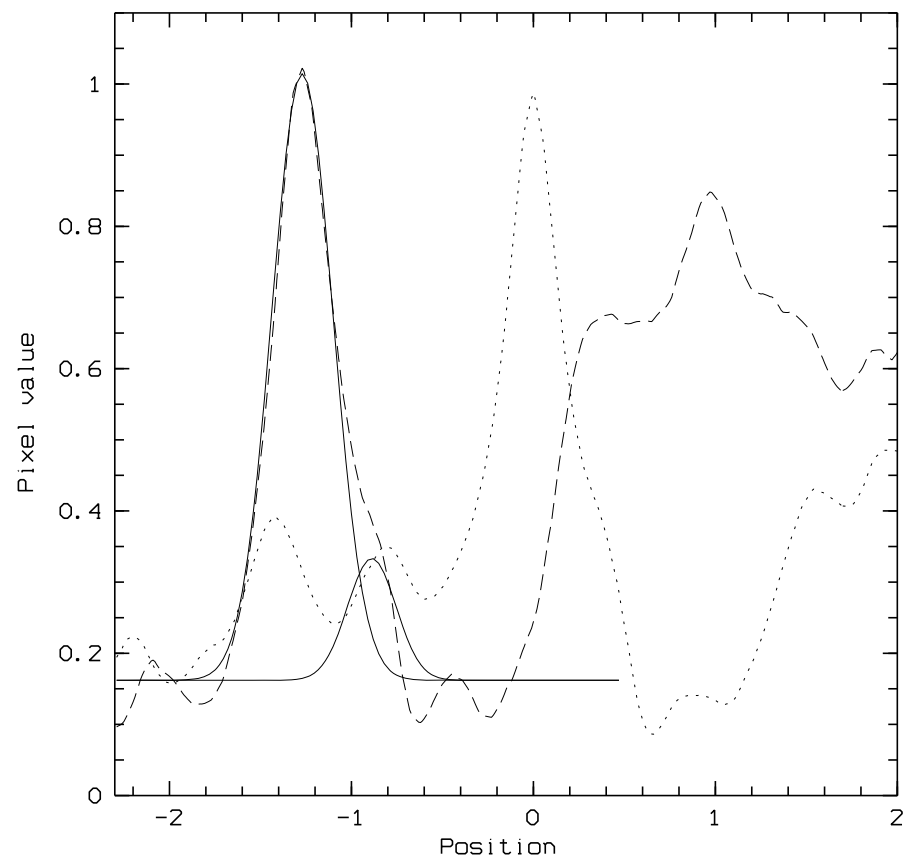

Fig. 12. We show here the cross-correlation profiles of Gl 488 with itself (dotted line) and of McC 332 with Gl 488 (dashed line). The two Gaussian fit of the latter profile is also shown.

Table 2. Summary of the two Gaussian fits to the correlation profile and the CaII $\mathrm{H}$ line.

\begin{tabular}{ccccccc}
\hline \hline $\begin{array}{c}\text { Profile } \\
\text { Type }\end{array}$ & $\begin{array}{c}\lambda_{0} \\
(\AA)\end{array}$ & $\begin{array}{c}F W H M \\
(\AA)\end{array}$ & $\begin{array}{c}\text { Max } \\
\text { flux }\end{array}$ & $\begin{array}{c}\lambda_{0} \\
(\AA)\end{array}$ & $\begin{array}{c}F W H M \\
(\AA)\end{array}$ & $\begin{array}{c}\text { Max } \\
\text { flux }\end{array}$ \\
\hline Correlation & -1.27 & 0.395 & 0.880 & -0.888 & 0.314 & 0.17 \\
CaII H & 3967.04 & 0.24 & 0.879 & 3967.26 & 0.23 & 2.96 \\
\hline
\end{tabular}

the finding that McC 332 is a binary. The wavelength shift yields a velocity of $9 \mathrm{~km} \mathrm{~s}^{-1}$.

The CaII H spectrum of McC 332 is shown in Fig. 14. We can see a clear asymmetry in this profile. The profile fitted by two Gaussians yields a wavelength difference of $0.22 \AA$, i.e., $16.6 \mathrm{~km} \mathrm{~s}^{-1}$. Note that the $\mathrm{H}_{\alpha}$ and CaII $\mathrm{H}$ lines were not observed at the same time. We obtain a mean redshift for the secondary component of about $14 \mathrm{~km} \mathrm{~s}^{-1}$. Note that the CaII H line is stronger for the secondary than for the primary. The parameters of our two-Gaussian fit are summarized in Table 2.

\section{Conclusion}

We have observed a number of spectral lines for a few $\mathrm{dK}$, $\mathrm{dM}$ and $\mathrm{dMe}$ stars that provide new constraints for the purpose of NLTE-radiation transfer modelling of their chromospheres. Notably, $\mathrm{Pa}_{\epsilon}$ was observed for the first time. We plan to use these data to build new model chromospheres. The Paschen lines are complementary to the Balmer lines as they are formed lower in the chromosphere (Short \& Doyle 1998a). It would be interesting to reconcile the modelling of these lines, especially since Short \& Doyle (1998a) could not model both the Paschen and Balmer lines at the same time. With our detection of $\mathrm{Pa}_{\epsilon}$ we also have better constraints than those of Short \& Doyle (1998a) who barely detected the $\mathrm{Pa}_{\beta}$ line.

We have gathered complete sets of observations of chromospheric lines from the lower chromosphere to the transition region. These sets are unique and are precious to develop 


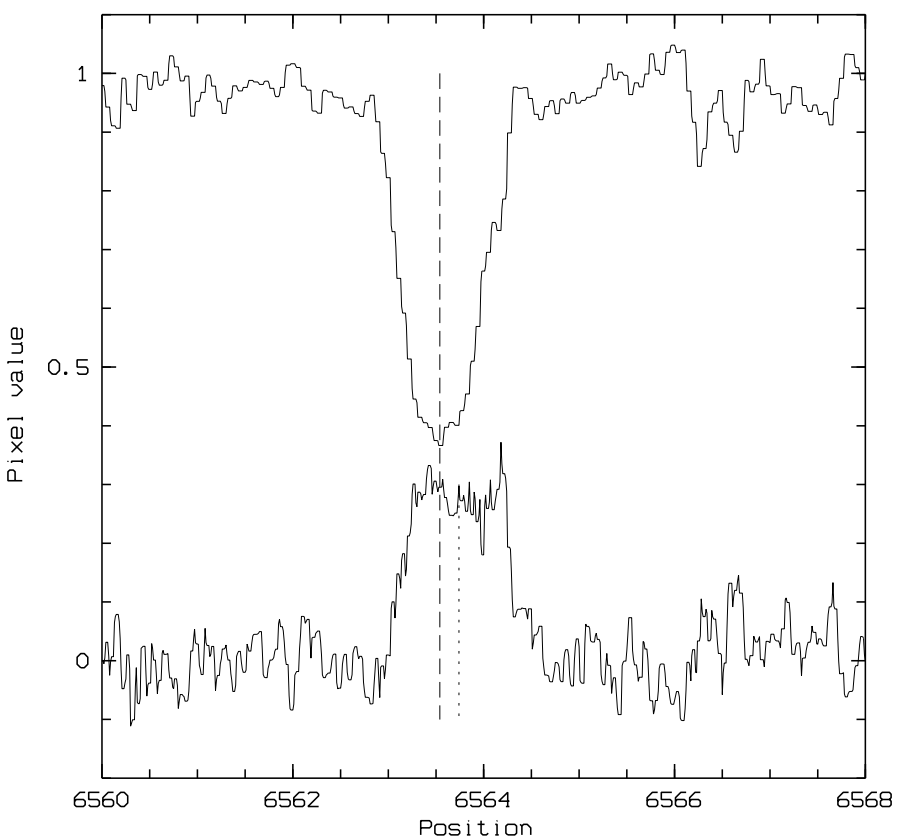

Fig. 13. We show here the difference spectrum McC 332-Gl 488, together with the spectrum of Gl 488. Note the redshift of the emission profile in the difference spectrum.

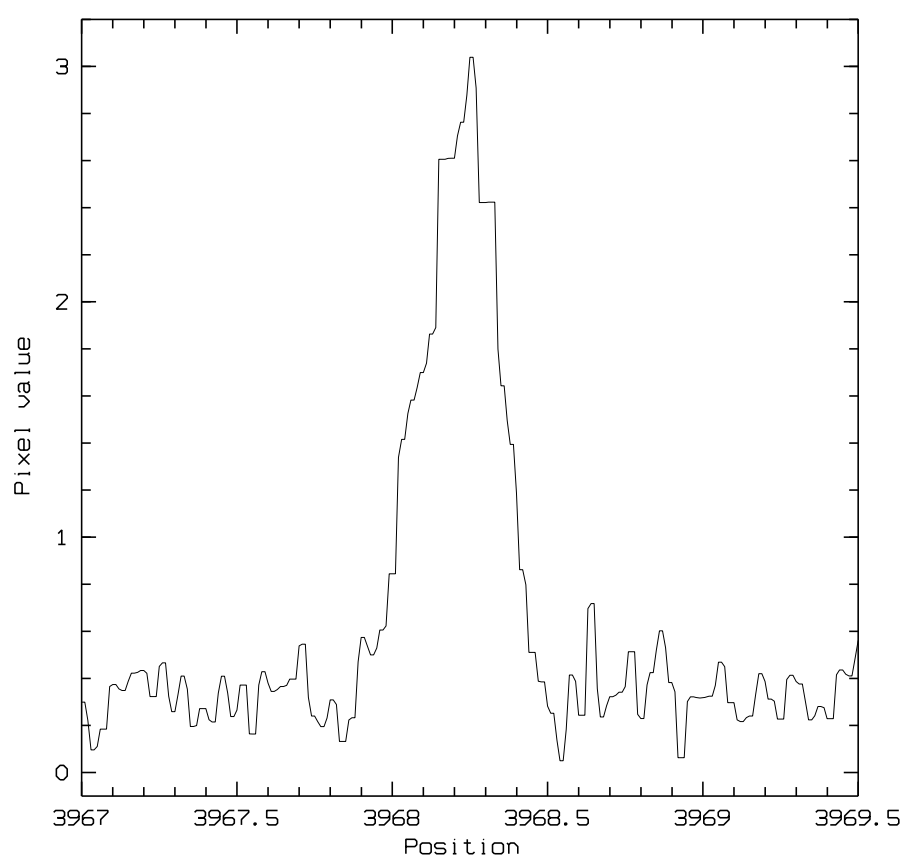

Fig. 14. We show here the CaII H profile of McC 332. Note the asymmetry of this line and the redshift of the strongest emission profile.

complete model chromospheres. So far, very few studies have attempted to model such a large number of spectral lines. We will attempt to model these spectral features with two-component model chromospheres with the help of grids of model chromospheres (Papers VI, XI). This will be a substantial improvement over previous studies which often analyzed the formation of one spectral feature at a time (see references therein). AU Mic is of particular interest as well as G1 729, and we plan to improve the AU Mic model developed in Paper II. Of particular interest are also the differences in the spectra of AU Mic and G1 729. Modelling will have to explain these important differences.
We have observed a bright spectroscopic binary, composed of a dK7 primary and a dM3.5e secondary. The secondary is quite active with strong $\mathrm{H}_{\alpha}$ and CaII $\mathrm{H}$ emissions. We plan to gather more such high resolution observations of $\mathrm{K}$ and $\mathrm{M}$ dwarfs in order to build more accurate grids of model chromospheres and for different spectral types from dK5 to dM8.

Acknowledgements. The authors would like to thank Dr. C. J. Butler from Armagh Observatory for improving the English of this manuscript.

\section{References}

Andretta, V., Doyle, J. G., \& Byrne, P. B. 1997, A\&A, 322, 266

Ayres, T. R. 1979, ApJ, 228, 509

Ayres, T. R., Eriksson, K., Linsky, J. L., \& Stencel, R. E. 1983, ApJ, 270, L17

Balega, I., Balega, Y. Y., Maksimov, A. F., et al. 2004, A\&A, 422, 627

Baliunas, S. L. 1981, Cool Stars, Stellar Systems and the Sun, ed. M. S. Giampapa, \& L. Golub, SAO Special Report, No. 392, II, 31

Baliunas, S. L., Vaughan, A. H., Hartmann, L., et al. 1983, ApJ, 275, 752

Baliunas, S. L., Donahue, R. A., Soon, W. H., et al. 1995, ApJ, 438, 269

Baranovskii, E. A., Gershberg, R. E., \& Shakhovskoi, D. N. 2001, Astron. Rep., 45, 67

Bessel, M. S. 1990, A\&AS, 83, 357

Blanco, C., Catalano, S., Marilli, E., \& Rodonò, M. 1974, A\&A, 33, 257

Bonfils, X., Delfosse, X., Udry, S., et al. 2005, A\&A, 442, 635

Brosche, P. 1960, Astron. Nachr., 285, 261

Busa, I., Andretta, V., Gomez, M. T., \& Terranegra, L. 2001, A\&A, 373, 993

Butler, C. J., Byrne, P. B., Andrews, A. D., \& Doyle, J. G. 1981, MNRAS, 197, 815

Butler, C. J., Doyle, J. G., Andrews, A. D., et al. 1987, A\&A, 174, 139

Byrne, P. B. 1993, A\&A, 272, 495

Byrne, P. B., \& Doyle, J. G. 1989, A\&A, 208, 159

Byrne, P. B., \& Doyle, J. G. 1990, A\&A, 238, 221

Cayrel de Strobel, G., Perrin, M. N., Cayrel, R., \& Lebreton, Y. 1989, A\&A, 225, 369

Cenarro, A. J., Peletier, R. F., Sanchez-Blazquez, P., et al. 2007, MNRAS, 374, 664

Cincunegui, C., Diaz, R. F., \& Mauas, P. J. D. 2007, A\&A, 469, 309

Cram, L. E., \& Mullan, D. J. 1979, ApJ, 234, 579

Cram, L. E., \& Mullan, D. J. 1985, ApJ, 294, 626

Cram, L. E., \& Giampapa, M. S. 1987, ApJ, 323, 316

Cristaldi, S., \& Rodonò, M. 1970, A\&AS, 2, 223

Cuntz, M., Rammacher, W., Ulmschneider, P., Musielak, Z. E., \& Saar, S. H. 1999, ApJ, 522, 1053

Cutispoto, G., \& Giampapa, M. S. 1988, PASP, 100, 1452

Del Zanna, G., Landini, M., \& Mason, H. E. 2002, A\&A, 385, 968

Dempsey, R. C., Neff, J. E., \& Lim, J. 2001, ApJ, 122, 332

Diaz, R. F., Cincunegui, C., \& Mauas, P. J. D. 2007, MNRAS, 378, 1007

Doyle, J. G. 1987, MNRAS, 224, Short Communication 1

Doyle, J. G., Panagi, P., \& Byrne, P. B. 1990, A\&A, 288, 443

Doyle, J. G., Houdebine, E. R., Mathioudakis, M., \& Panagi, P. 1994, A\&A, 285, 233

Doyle, J. G., Mathioudakis, M., Andretta, V., Short, C. I., \& Jelinsky, P. 1997, A\&A, 318, 835

Elgaroy, O., Engvold, O., \& Lund, N. 1999, A\&A, 343, 222

Fekel, F. C. 1997, PASP, 109, 514

Fitzgerald, M. P., Kalas, P. G., Duchene, G., Pinte, C., \& Graham, J. R. 2007, ApJ, 670, 536

Fuhrmeister, B., Schmitt, J. H. M. M., \& Hauschildt, P. H. 2005, A\&A, 439, 1137

Gayley, K. G. 1994, ApJ, 431, 806

Gershberg, R. E., Katsova, M. M., Lovkaya, M. N., Terebzh, A. V., \& Shakovskaya, N .I. 1999, A\&AS, 139, 555

Giampapa, M. S., Worden, S. P., Schneeberger, T. J., \& Cram, L. E. 1981, ApJ, 246,502

Giampapa, M. S., Worden, S. P., \& Linsky, J. L. 1982, ApJ, 258, 740

Gray, R. O., Corbally, C. J., Garrison, R. F., et al. 2006, PJ, 132, 161

Hawley, S. L., Gizis, J. E., \& Reid, I.N. 1996, AJ, 112, 2799

Henry, T. J., Soderblom, D. R., Donahue, R. A., \& Baliunas, S. L. 1996, AJ, 111, 439

Herbst, W., \& Layden, A. C. 1987, AJ, 94, 150

Herbst, W., \& Miller, J. R. 1989, AJ, 97, 891

Houdebine, E. R. 1990, Ph.D. dissertation of the University of Orsay-Paris XI Houdebine, E. R. 1994, in the Proceedings of the 4th Workshop on Multi-Site Continuous Spectroscopy help at Beijing, China, ed. L. Huang et al., 179 Houdebine, E. R. 2008, MNRAS, 390, 1081 
Houdebine, E. R. 2009a, A\&A, submitted (Paper X)

Houdebine, E. R. 2009b, A\&A, in press (Paper XI)

Houdebine, E. R. 2009c, MNRAS, in press (Paper XII)

Houdebine, E. R. 2009d, MNRAS, in press (Paper XIII)

Houdebine, E. R., \& Doyle, J. G. 1994a, A\&A, 289, 169 (Paper I)

Houdebine, E. R., \& Doyle, J. G. 1994b, A\&A, 289, 185 (Paper II)

Houdebine, E. R., \& Doyle, J. G. 1995, A\&A, 302, 861 (Paper IV)

Houdebine, E. R., \& Stempels, H. C. 1997, A\&A, 326, 1143 (Paper VI)

Houdebine, E. R., \& Panagi, P. M. 1990, A\&A, 231, 459

Houdebine, E. R., Doyle, J. G., \& Kościelecki, M. 1995, A\&A, 294, 773 (Paper III)

Houdebine, E. R., Mathioudakis, M., Doyle, J. G., \& Foing, B. H. 1996, A\&A, 305, 209 (Paper V)

Hunsch, M., Schmitt, J. H. M. M., Sterzik, M. F., \& Voges, W. 1999, A\&AS, 135,319

Jenkins, J. S., Jones, H. R. A., Tinney, C. G., et al. 2008, MNRAS, 372, 163

Jevremovic, D., Doyle, J. G., \& Short, C. I. 2000, A\&A, 358, 575

Johns-Krull, C. M., \& Valenti, J. A. 1996, ApJ, 459, L95

Kamper, K. W., Kang, M. K., \& Thomson, J. R. 1997, Baltic Astron., 6, 111

Kelch, W. L., Linsky, J. L., \& Worden, S. P. 1979, ApJ, 229, 700

Kunkel, W. E. 1973, ApJS, 25, 1

Kraft, R. P., Preston, G. W., \& Wolff, S. C. 1964, ApJ, 136, 793

Landsman, W., \& Simon, T. 1993, ApJ, 408, 305

Lanzafame, A. C. 1995, A\&A, 302, 839

Lanzafame, A. C., \& Byrne, P. B. 1995, A\&A, 303, 155

Linsky, J. L., \& Wood, B. E. 1994, ApJ, 430, 342

Linsky, J. L., Bornmann, P. L., Carpenter, K. G., et al. 1982, ApJ, 260, 670

Lopez-Morales, M. 2007, ApJ, 660, 732

Maran, S. P., Robinson, R. D., Shore, S. N., et al. 1994, ApJ, 421, 800

Marcy, G. W., \& Chen, G. H. 1992, ApJ, 390, 550

Mathioudakis, M., \& Doyle, J. G. 1989, A\&A, 224, 179

Mathioudakis, M., \& Doyle, J. G. 1990, A\&A, 240, 357

Mathioudakis, M., \& Doyle, J. G. 1991, A\&A, 244, 409

Mathioudakis, M., \& Doyle, J. G. 1992, A\&A, 262, 523

Mauas, P. J. D., \& Falchi, A. 1994, A\&A, 281, 129

Mauas, P. J. D., Falchi, A., Pasquini, L., \& Pallavicini, R. 1997, A\&A, 326, 249

Monsignori Fossi, B. C., \& Landini, M. 1994, A\&A, 284, 900

Monsignori Fossi, B. C., Landini, M., Del Zanna, G., \& Bowyer, S. 1996, ApJ, 466, 427

Noyes, R. W., Hartmann, L. W., Baliunas, S. L., Duncan, D. K., \& Vaughan, A. H. 1984, ApJ, 279, 763

Oranje, B. J. 1986, A\&A, 154, 185

Osten, R. A., Ayres, T. R., Brown, A., Linsky, J. L., \& Krishnamurthi, A. 2003, ApJ, 582, 1073

Osten, R. A., Hawley, S. L., Allred, J., et al. 2006, ApJ, 647, 1349

Pagano, I., Linsky, J. L., Carkner, L., et al. 2000, ApJ, 532, 497
Panagi, P. M., \& Mathioudakis, M. 1993, A\&AS, 100, 343

Pasquini, L., \& Pallavicini, R. 1991, A\&A, 251, 199

Pettersen, B. R. 1989, A\&A, 209, 279

Poveda, A., Herrera, M. A., Allen, C., Cordero, G., \& Lavalley, C. 1994, RMAA, 28,43

Quin, D. A., Doyle, J. G., Butler, C. J., Byrne, P. B., \& Swank, J. H. 1993, A\&A, 272,477

Rauscher, E., \& Marcy, G. W. 2006, PASP, 118, 617

Redfield, S., \& Linsky, J. L. 2002, ApJS, 139, 439

Reid, I. N., Hawley, S. L., \& Gizis, J. E. 1995, AJ, 110, 1838

Robinson, R. D., Cram, L. E., \& Giampapa, M. S. 1990, ApJS, 74, 891

Rutten, R. G. M., Schrijver, C. J., Zwaan, C., Duncan, D. K., \& Mewe, R. 1989, A\&A, 219, 239

Saar, S. H., \& Osten, R. A. 1997, MNRAS, 284, 803

Saar, S. H., Huovelin, J., Osten, R. A., \& Shcherbakov, A. G. 1997, A\&A, 326, 741

Shara, M. M., Bergeron, L. E., Christian, C. A., Craig, N., \& Bowyer, S. 1997, PASP, 109, 998

Schrijver, C. J., Mewe, R., van den Oord, G. H. J., \& Kaastra, J.S. 1995, A\&A, 302,438

Segransan, D., Kervella, P., Forveille, T., \& Queloz, D. 2003, A\&A, 397, L5

Scoville, F., \& Mena-Werth, J. 1998, PASP, 110, 794

Short, C. I., \& Doyle, J. G. 1997, A\&A, 326, 287

Short, C. I., \& Doyle, J. G. 1998a, A\&A, 331, L5

Short, C. I., \& Doyle, J. G. 1998b, A\&A, 336, 613

Short, C. I., Doyle, J. G., \& Byrne 1997, A\&A, 324, 196

Sim, S. A., \& Jordan, C. 2005, MNRAS, 361, 1102

Stauffer, J. R., \& Hartmann, L. W. 1986, ApJS, 61, 531

Tinney, C. G., McCarthy, C., Jones, H. R. A., et al. 2002, MNRAS, 332, 759

Tripicchio, A., Severino, G., Covino, E., Terranegra, L., \& Garcia Lopez, R. J. 1997, A\&A, 327, 681

Tripicchio, A., Gomez, M. T., Severino, G., et al. 1999, A\&A, 345, 915

Vaughan, A. H., Baliunas, S. L., Middelkoop, F., et al. 1981, ApJ, 250, 276

Walkowicz, L. M. 2008, Ph.D. Thesis dissertation of the University of Washington, Self-consistent quiescent model atmospheres for M dwarfs Walkowicz, L. M., \& Hawley, S. L. 2008, AJ, 137, 3297

Walkowicz, L. M., \& Hawley, S. L. 2009, in the Proceedings of Cool Stars, Stellar Systems and the Sun, AIP Conf. Proc., 1094, 696

Weis, E. W. 1993, AJ, 105, 1962

Wilson, O. C., \& Bappu, M. K. 1957, ApJ, 125, 661

Wilson, O., \& Wooley, R. 1970, MNRAS, 148, 463

Wood, B. E., Linsky, J. L., \& Ayres, T. R. 1997, ApJ, 478, 745

Worden, S. P., Schneeberger, T., \& Giampapa, M. S. 1981, ApJS, 46, 159

Wright, J. T., Marcy, G. W., Butler, R. P., \& Vogt, S. S. 2004, ApJS, 152, 261

Young, A., Skumanich, A., Stauffer, J. R., Bopp, B. W., \& Harlan, E. 1989, ApJ, 344,427 